\title{
New horizons in cosmology with spectral distortions of the cosmic microwave background
}

\author{
J. Chluba' ${ }^{1}$ - M. H. Abitbol ${ }^{2}$ - N. Aghanim ${ }^{3}$ - Y. Ali-Haïmoud ${ }^{4}$ • M. Alvarez ${ }^{5,6}$. \\ ${\mathrm{K} . \text { Basu }^{7} \text { - B. Bolliet }}^{1}$ - C. Burigana ${ }^{8,9,10}$ - P. de Bernardis ${ }^{11,12}$. \\ J. Delabrouille $\mathrm{e}^{13,14}$. E. Dimastrogiovanni ${ }^{15}$. F. Finelli ${ }^{16}$. D. Fixsen ${ }^{17}$. \\ L. Hart ${ }^{1}$. C. Hernández-Monteagudo ${ }^{18}$. J. C. Hill ${ }^{19,20}$ - A. Kogut ${ }^{21}$. \\ K. Kohri ${ }^{22}$ - J. Lesgourgues ${ }^{23}$ - B. Maffei ${ }^{3}$. J. Mather ${ }^{21}$. S. Mukherjee 24,25,26 . \\ S. P. Patil ${ }^{27}$ - A. Ravenni ${ }^{1}$ - M. Remazeilles ${ }^{1}$ - A. Rotti ${ }^{1}$ - J. A. Rubiño-Martin ${ }^{28,29}$. \\ J. Silk 30,31 • R. A. Sunyaev $19,32,33$ - E. R. Switzer ${ }^{21}$
}

Received: 28 July 2020 / Accepted: 3 March 2021 / Published online: 6 May 2021

(C) The Author(s) 2021

\begin{abstract}
This Voyage 2050 paper highlights the unique science opportunities using spectral distortions of the cosmic microwave background (CMB). CMB spectral distortions probe many processes throughout the history of the Universe, delivering novel information that complements past, present and future efforts with CMB anisotropy and large-scale structure studies. Precision spectroscopy, possible with existing technology, would not only provide key tests for processes expected within the cosmological standard model but also open an enormous discovery space to new physics. This offers unique scientific opportunities for furthering our understanding of inflation, recombination, reionization and structure formation as well as dark matter and particle physics. A dedicated experimental approach could open this new window to the early Universe in the decades to come, allowing us to turn the long-standing upper distortion limits obtained with $C O B E$ /FIRAS some 25 years ago into clear detections of the expected standard distortion signals and also challenge our current understanding of the laws of nature.
\end{abstract}

Keywords Cosmology $\cdot$ Early universe $\cdot$ Cosmic microwave background $\cdot$ CMB spectral distortions $\cdot$ Particle physics

For endorsement and a broader overview of all the related microwave spectro-polarimetric papers please visit here: www.microwave-spectropolarimetry.eu.

J. Chluba

jens@chluba.de

Extended author information available on the last page of the article. 


\section{Introduction}

Following the pioneering observations with $C O B E$ in the early 1990 s, studies of the cosmic microwave background (CMB) have primarily focused on temperature and polarization anisotropies. $\mathrm{CMB}$ spectral distortions - tiny departures of the CMB energy spectrum from that of a perfect blackbody - provide a second, independent probe of fundamental physics, with a reach deep into the primordial Universe. The theoretical foundation of spectral distortions has seen major advances in recent years, highlighting the immense potential of this emerging field. Spectral distortions probe a fundamental property of the Universe - its thermal history - thereby providing additional insight into processes within the cosmological standard model ${ }^{1}$ (CSM) as well as new physics beyond. Spectral distortions are an important tool for understanding inflation and the nature of dark matter. They shed new light on the physics of recombination and reionization, both prominent stages in the evolution of our Universe, and furnish critical information on baryonic feedback processes, in addition to probing primordial correlation functions at scales inaccessible to other tracers. In principle the range of signals is vast: many orders of magnitude of discovery space can be explored by detailed observations of the CMB energy spectrum. Several CSM signals are predicted and provide clear experimental targets that are observable with present-day technology. Confirmation of these signals would extend the reach of the CSM by orders of magnitude in physical scale as the Universe evolves from the initial stages to its present form. Their absence would pose a huge theoretical challenge, immediately pointing to new physics.

Here, we advocate for a dedicated effort to measure CMB spectral distortions at the largest angular scales $\left(\gtrsim 1^{\circ}\right)$ within the ESA Voyage 2050 program (see Section 4.3 for roadmap). We argue that an L-class mission with a pathfinder would allow a precise measurement of all the expected CSM distortions. With an M-class mission, the primordial distortions (created at $z \gtrsim 10^{3}$ ) would still be detected at modest significance, while the late-time distortions will continue to be measured to high accuracy. Building on the heritage of $C O B E$ /FIRAS [1, 2], a spectrometer that consists of multiple, cooled $(\simeq 0.1 \mathrm{~K})$, absolutely-calibrated Fourier Transform Spectrometers (FTS) with wide frequency coverage $(v \simeq 10 \mathrm{GHz}$ to a few $\times \mathrm{THz})$ and all-sky spectral sensitivity at the level of $0.1-0.5 \mathrm{Jy} / \mathrm{sr}$ would be the starting point for the M-class option. A scaled and further optimized version of this concept is being envisioned as the L-class option. Such measurements can only be done from space and would deliver hundreds of absolutely-calibrated maps of the Universe at large scales, opening numerous science opportunities for cosmology and astrophysics (see Section 4.4 for synergies). This will provide independent probes of inflation, dark matter and particle physics, recombination and the energy output of our Universe from at late times, turning the long-standing spectral distortion limits of $C O B E /$ FIRAS into clear detections.

\footnotetext{
${ }^{1}$ When referring to the cosmological standard model (CSM) we assume the $\Lambda$ CDM parametrization, supplemented by the Standard Model of particle physics, admitting that the presence of dark matter and dark energy requires physics beyond the latter.
} 
This paper is structured as follows: in Section 2, we provide a brief introduction to the topic of CMB spectral distortions. This is then followed by an overview of various sources of spectral distortions within the CSM and beyond in Section 3. Some of the main scientific targets are summarized in Fig. 11 and discussed with respect to the experimental and observational challenges in Section 4. In Section 4.3, we explain the overall experimental roadmap, followed by a consideration of broader synergistic elements in Section 4.4. We then close the paper with a brief summary of the main conclusions in Section 5.

\section{Cosmology beyond thermal equilibrium}

Cosmology is now a precise scientific discipline, with a detailed theoretical model that fits a wealth of very accurate measurements. Of the many cosmological data sets, the $\mathrm{CMB}$ temperature and polarization anisotropies provide the most stringent and robust constraints, allowing us to determine the key parameters of our Universe (e.g., the total density, expansion rate and baryon content) with unprecedented precision, while simultaneously addressing fundamental questions about inflation and earlyUniverse physics. By studying the statistics of the CMB anisotropies with different experiments over the past decades we have entered the era of precision cosmology, clearly establishing the highly-successful $\Lambda \mathrm{CDM}$ concordance model [3-5].

But the quest continues. Despite its many successes, $\Lambda \mathrm{CDM}$ is known to be incomplete. It traces the growth of structure in the Universe from primordial density perturbations to the modern era, but the origin of those perturbations remains poorly understood. In addition, in spite of relentless efforts, the nature of dark matter (DM) and dark energy remains a mystery. Together, these enigmatic components comprise 95\% of the energy density of the Universe. Particle and high-energy physics offer candidate solutions for these problems (e.g., inflation and particle dark matter), but these inevitably require new physics beyond the Standard Model of particle physics.

Precision measurements of the $\mathrm{CMB}$ energy spectrum open a new window into the physics of the early Universe, constraining cosmological models in ways not possible using other techniques. Departures of the CMB energy spectrum from a pure blackbody - commonly referred to as spectral distortions - encode unique information about the thermal history of the Universe, from when it was a few months old until today. Since the measurements with $C O B E$ /FIRAS in the early '90s, the sky-averaged CMB spectrum is known to be extremely close to a perfect blackbody at a temperature $T_{0}=(2.7255 \pm 0.0006) \mathrm{K}[2,6]$, with possible distortions limited to one part in $10^{5}$. This impressive measurement was awarded the 2006 Nobel Prize in Physics and already rules out cosmologies with extended periods of large energy release. Here we propose to revisit the measurement of the CMB spectrum with current and upcoming technology significantly advancing the existing frontier.

\subsection{Main types of spectral distortions}

Spectral distortions are created by processes that drive matter and radiation out of thermal equilibrium after thermalization becomes inefficient at redshift $z \lesssim 2 \times 10^{6}$. 
Examples are energy-releasing mechanisms that heat the baryonic matter or inject photons or other electromagnetically-interacting particles. The associated signals are usually characterized as $\mu$ - and $y$-type distortions, formed by energy exchange between electrons and photons through Compton scattering [8-12]. Compton scattering is inefficient at $z \lesssim 5 \times 10^{4}$, yielding a $y$-type distortion, which probes the thermal history during recombination and reionization (Fig. 1). In contrast, a $\mu$-type (or chemical potential) distortion forms at $z \gtrsim 5 \times 10^{4}$, when Compton scattering is very efficient in redistributing photons across energy. A $\mu$-distortion cannot be generated at recent epochs and thus directly probes events in the pre-recombination era.

The simple classical picture has been refined in recent years. We now understand that the transition between $\mu$ - and $y$-type distortions is gradual (see intermediate regime in Fig. 1 at redshifts $10^{4} \lesssim z \lesssim 3 \times 10^{5}$ ) and that the signal contains additional time-dependent information [13-15]. This extra information is contained in the residual or $r$-type distortion, which cannot be described by a simple sum of $\mu$ and $y$, and thus can be used to distinguish energy release mechanisms [16, 17].

It was also shown that distortions created by photon-injection mechanisms can exhibit a rich spectral phenomenology [18]. One prominent example is the distortion created by the cosmological recombination process [19-21] (see Fig. 1). Additional

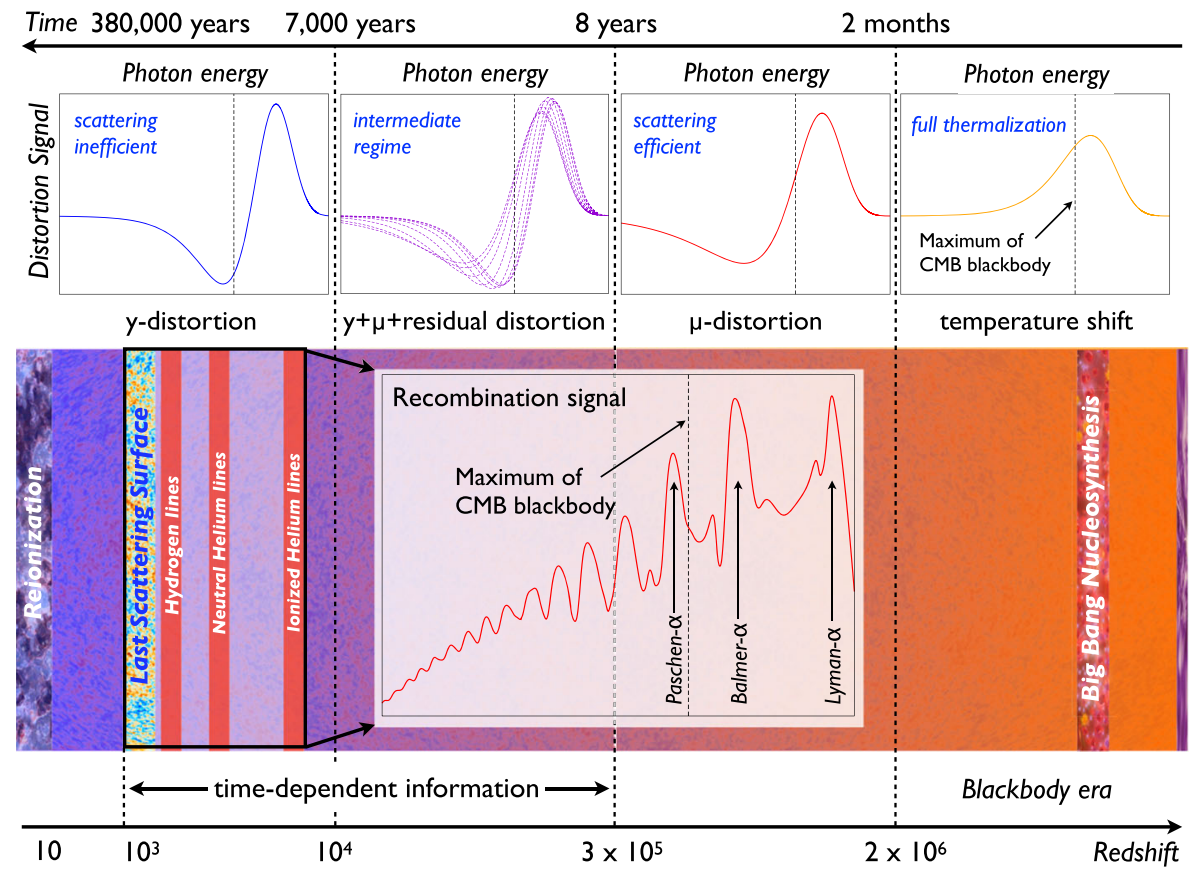

Fig. 1 Evolution of spectral distortions across time. Distortions probe the thermal history over long periods deep into the primordial Universe that are inaccessible by other means. The distortion shape contains valuable epoch-dependent information that allows distinguishing different sources of distortions. Lineemission is created during the cosmological recombination eras leaving a detailed 'fingerprint' of the recombination process. The figure is adapted from [7] 
epoch-dependent information can be imprinted by non-equilibrium processes in the pre-recombination hydrogen and helium plasma [22-24] or by non-thermal particles in high-energy particle cascades (e.g., [18, 24-27]).

Spectral distortions thus provide more than just a simple integral constraint for cosmology. They are a unique and powerful probe of a wide range of interactions between particles and $\mathrm{CMB}$ photons, reaching back all the way from the present to a few months after the Big Bang and allowing us to access information that cannot be extracted in any other way. Broad overviews of the CMB spectral distortion science case can be found in [13, 20, 28-36].

\subsection{CMB spectral distortion signals across the sky}

While CMB distortion signals can span a wide range of spectral shapes, another important way to distinguish them is through their distribution across the sky. CMB spectral distortions are usually isotropic signals, directly imprinted in the energy distribution of the sky-averaged CMB (i.e., the monopole). To extract spectral distortions one therefore has to measure the absolute photon flux at different frequencies, while the direction on the sky is secondary. This requires accurate absolute calibration $[1,37]$ or accurate channel inter-calibration [20, 38, 39], which can be achieved with experimental concepts like PIXIE [40, 41]. To minimize foreground contaminations, prior knowledge (e.g., from Planck) can be used to optimize the scanning strategy and beam size. This implies that spectral distortion measurements at large angular scales $\left(\gtrsim 1^{\circ}\right)$ are optimal.

However, CMB spectral distortions can also have anisotropic components. One prominent example is due to the Sunyaev-Zeldovich (SZ) effect [9, 42] caused by the scattering of photons by energetic electrons inside clusters of galaxies, which has become an important tool for cosmology (e.g., [43]). In contrast, anisotropic $\mu$ - or $y$-distortions from the pre-recombination era $\left(z>10^{3}\right)$ are expected to be negligible, can, however, be boosted to visible levels due to super-horizon mode correlations, e.g., caused by primordial non-Gaussianity [44-47] (see Section 3.2). The CMB dipole spectrum is furthermore distorted due to our motion with respect to the CMB restframe [48-50]. Additional distortions due to the observer's motion also appear in the higher multipoles [51]. Line and resonance scattering effects also leave anisotropic imprints (Sections 3.8 and 3.9). All these signals can be correlated against tracers of both primordial density perturbations and large-scale structure to further probe cosmic evolution [44, 45, 52-56]. Lastly, measurements of distortion anisotropies may also shed new light on the origin of the large-scale CMB anomalies [57].

The focus of this paper is to optimize towards measurements of distortions signals at large angular scales $\left(\gtrsim 1^{\circ}\right)$, primarily targeting the monopole signals. A more detailed discussion of possible experimental concepts is left to Section 4; however, in the following, PIXIE [40, 41] and an enhanced version, SuperPIXIE [58], will be used as benchmarks. In the presence of foregrounds, PIXIE could reach one standard deviation errors of $\sigma(y) \simeq 3.4 \times 10^{-9}$ and $\sigma(\mu) \simeq 3 \times 10^{-8}$, while SuperPIXIE could acheive $\sigma(y) \simeq 1.6 \times 10^{-9}$ and $\sigma(\mu) \simeq 7.7 \times 10^{-9}$ (see Section 4.2, Fig. 10). Both concepts would improve the long-standing distortion limits of COBE/FIRAS 
$|y|<1.5 \times 10^{-5}$ and $|\mu|<9 \times 10^{-5}$ (95\% c.l.) [1,2] by several orders of magnitude. A dedicated high-resolution CMB imager approach is discussed in [59] and could enable precise spectral measurements at small angular scales, targeting SZ clusters, high- $\ell$ line-scattering signals and other secondary CMB anisotropies.

Finally, we mention that polarized CMB spectral distortions are usually negligible, such that one can in principle focus on intensity measurements only. However, polarization sensitivity could be useful for component separation. It was furthermore demonstrated that polarization-sensitive spectrometers like PIXIE [40, 41] could place tight constraints on the tensor-to-scalar ratio, $r$, reaching $\sigma(r) \simeq 10^{-3}[40$, 41], and could deliver a cosmic-variance-limited measurement of the Thomson optical depth, $\tau$ [60], to complement future ground-based experiments in their efforts to measure neutrino masses (e.g., CMB-S4). Polarization capabilities should thus still be considered when designing future $\mathrm{CMB}$ spectrometers.

\section{Spectral distortions as novel tests of $\Lambda$ CDM and beyond}

\subsection{CMB spectral distortions as a probe of inflation physics}

A central question in modern cosmology is the origin of the observed primordial density perturbations. Measurements from CMB anisotropies and large-scale structure find a nearly scale-invariant power spectrum $\mathcal{P}(k) \simeq k^{n \mathrm{~s}^{-1}}$ with spectral index $n_{\mathrm{S}}=0.965 \pm 0.004$, sampled over a range of spatial scales $k \simeq 10^{-4}$ to $0.1 \mathrm{Mpc}^{-1}$ [61]. Their phase coherence is an indication of their super-Hubble nature, and their near scale-invariance is evidence of a weakly broken shift symmetry in the underlying theory. However, their precise origin is as of yet unknown.

Inflation provides a widely accepted framework for generating these initial fluctuations [62-65], with the simplest models generically predicting a small departure from scale-invariance (with $n_{\mathrm{S}}<1$ ) as the inflaton rolls down its potential [66-69]. However, various alternatives to inflation have been proposed [70-78] and no clear theoretical consensus has yet emerged. Searches for primordial $B$-mode patterns in CMB polarization could yield additional evidence for the simplest inflationary models. So far CMB polarization measurements only provide upper limits $[61,79]$ with no firm target from theory for a guaranteed detection. However, detection of a tensor to scalar ratio of $r \simeq 10^{-3}$ is a distinguishing benchmark for large-field models, which in certain realizations further manifest the specific relation $r \simeq\left(1-n_{\mathrm{S}}\right)^{2}$ (e.g., $[62,80])$.

Spectral distortions provide a unique new probe of primordial density perturbations. Inflation may or may not be a valid description of the early Universe, but density perturbations are known to exist; regardless of their origin, dissipation of these perturbations through photon diffusion ( $\leftrightarrow$ Silk damping) in the early Universe will distort the CMB spectrum at observable levels [83-87]. The signal $(\mu+y+r-$ distortion) can be accurately calculated using simple linear physics and depends on the amplitude of primordial perturbations at scales with wavenumbers $k \simeq 1-$ $10^{4} \mathrm{Mpc}^{-1}$, some ten e-folds further than what can be probed by CMB anisotropies 


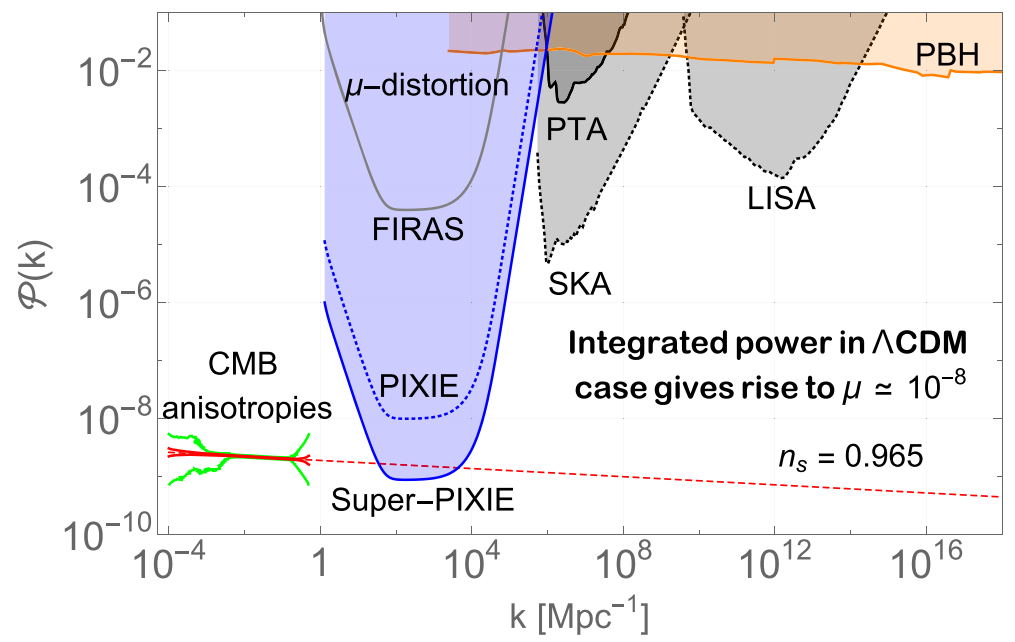

Fig. 2 Forecast constraints ( $95 \%$ c.1.) on the primordial power spectrum for features with a $k^{4}$ profile that cuts off sharply at some larger wavenumber $k_{p}$ (see [81], for more details). $\mu$-distortions constrain perturbations at scales and levels inaccessible to other probes. Early-Universe models with enhanced small-scale power at $k \simeq 10-10^{4} \mathrm{Mpc}^{-1}$ will be immediately ruled out if no distortion with $\mu>2 \times 10^{-8}$ is detected. The figure is adapted from [82]

(Fig. 2). ${ }^{2}$ Given an initial curvature power spectrum, $P(k)=2 \pi^{2} k^{-3} \mathcal{P}(k)$, the average $\mu$-distortion can be estimated with (e.g., [88, 89]):

$$
\langle\mu\rangle \approx \int \frac{k^{2} \mathrm{~d} k}{2 \pi^{2}} P(k) W_{\mu}(k),
$$

using an appropriate $k$-space window function, $W_{\mu}(k)$, which receives most of its contributions from modes with $k \simeq 10^{2} \mathrm{Mpc}^{-1}-10^{4} \mathrm{Mpc}^{-1}$. If the near scaleinvariance of the power spectrum observed on large scales persists to these much smaller scales, then the predicted distortion, $\mu \simeq(2.3 \pm 0.14) \times 10^{-8}[35,86$, 90], could be observed using current technology (Section 4). Detecting this signal extends our grasp on primordial density perturbations by over three orders of magnitude in scale, covering epochs that cannot be probed directly in any other way. $A$ non-detection at this level would be a serious challenge for $\Lambda \mathrm{CDM}$, immediately requiring new physics.

Measurements of $\mu$-distortions are directly sensitive to the power spectrum amplitude and its scale-dependence around $k \simeq 10^{3} \mathrm{Mpc}^{-1}$ [17, 88, 91, 92]. Within the slow-roll paradigm, this provides a handle on higher-order slow-roll parameters (often parametrized as running of the tilt or running of the running), benefiting from a vastly extended lever arm [17, 93-95]. Outside of standard slow-roll inflation, large

\footnotetext{
${ }^{2}$ To avoid the unrealistic GW spectrum generated by a $\delta$-function scalar power spectrum, we plot all integrated constraints using a $k^{4}$ spectrum - see [81] for the reason for this choice. The peak sensitivity for $\mu$-distortions is effectively unchanged were we to instead plot constraints for $\delta$-function features in the power spectrum with the same integrated power (see Fig. 9 therein), and also [96].
} 
departures from scale-invariance are well-motivated and often produce excess smallscale power (e.g., features [97-99] or inflection points [100-105] in the potential, particle production [106-110], waterfall transitions [111-115], axion inflation [116118], etc. [89]), implying the presence of new physical scales that can be probed with spectral distortions (Fig. 2). In this respect, a non-detection of the predicted $\mu$ distortions could establish a link to a possible primordial origin of the small-scale structure crisis [119-121]. Spectral distortions are also created by the dissipation of small-scale tensor perturbations [122, 123] and depend on the perturbation-type (i.e., adiabatic vs. iso-curvature) [124-127], providing additional ways to test inflation scenarios in uncharted territory.

Working within the slow-roll inflation paradigm, in Fig. 3, we further illustrate the gains in estimating the running of the spectral index when combining CMB anisotropy measurements with a CMB spectrometer. Due to the extended lever arm, small changes in $n_{\text {run }}$ significantly affect the $\mu$-distortion amplitude (see [86], for illustrations and approximations). Thus, CMB spectral distortion measurements allow improving constraints on $n_{\text {run }}[17,92-95,128]$. The expected improvement is larger for $n_{\text {run }}>0$, while it is lower for $n_{\text {run }}<0$, due to a reduced value of $\mu$ [17].

For standard slow-roll inflation models, $n_{\text {run }}$ is very close to zero, consistent with current best constraints from Planck: $n_{\text {run }}=-0.0041 \pm 0.0067$ [61]. Assuming a fiducial value of $n_{\text {run }}=0$ and combining Planck with a future spectrometer could tighten the error on $n_{\text {run }}$ by a factor of $\simeq 1.7$ to $\sigma\left(n_{\text {run }}\right) \simeq 0.004$ if a distortion sensitivity $\sigma(\mu) \simeq 5 \times 10^{-9}$ is achieved (see Fig. 3). To reach $\sigma\left(n_{\text {run }}\right) \simeq$

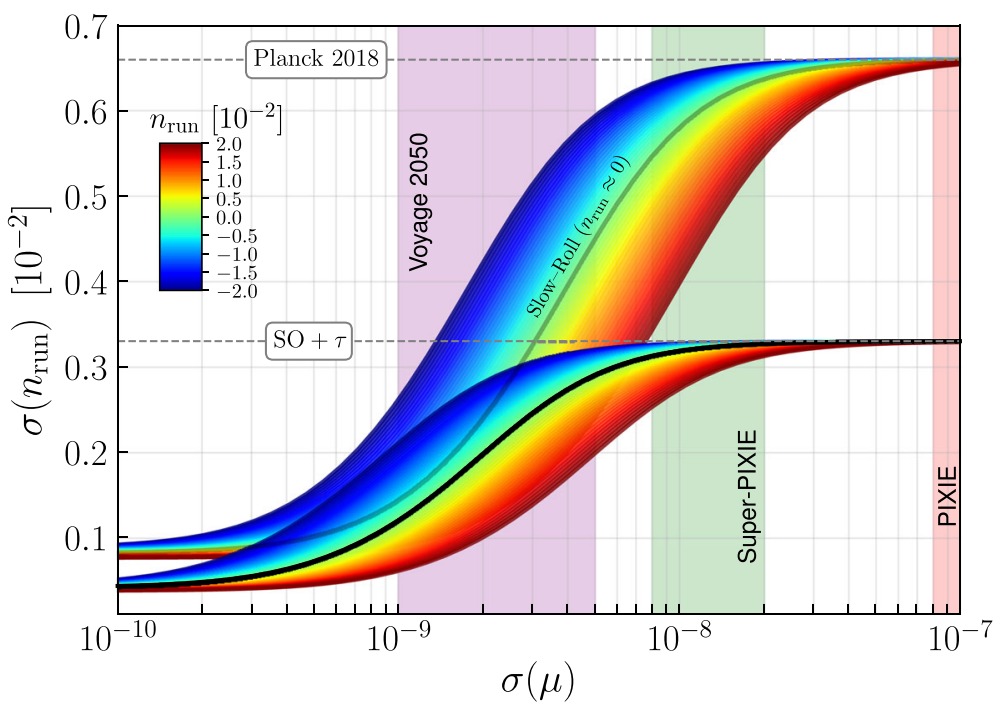

Fig. 3 Expected sensitivity to running of the spectral index, $n_{\text {run }}$, when combining CMB anisotropy measurements with a spectrometer of varying sensitivity to $\mu$. Across the colored band, the fiducial value for $n_{\text {run }}$ is varied. In all cases, a spectrometer leads to improvements of the constraint once $\sigma(\mu) \simeq 2 \times 10^{-8}$ can be reached. The improvement depends on the fiducial value of $n_{\text {run }}$. For reference, the case $n_{\text {run }}=0$ as expected from generic slow-roll scenarios is shown as solid black line. The shaded regions give plausible ranges for $\sigma(\mu)$ expected for the annotated concepts (Section 4.2) 
0.0033, as plausible for the funded Simons Observatory (SO) together with a cosmicvariance-limited measurement of $\tau$ [129], in combination with Planck one requires $\sigma(\mu) \simeq 3 \times 10^{-9}$. A cosmic-variance-limited measurement of $\tau$ itself is possible with a polarizing spectrometer, yielding $\sigma(\tau) \simeq 0.002$ [60], but is also expected to become available with Litebird [130]. By combining a spectrometer with ${ }^{3} \mathrm{SO}+\tau$, at $\sigma(\mu) \simeq 2 \times 10^{-9}$ we can further improve the error on $n_{\text {run }}$ to $\sigma\left(n_{\text {run }}\right) \simeq 0.002$, another factor of $\simeq 1.7$ better than $\mathrm{SO}+\tau$ alone. This highlights some of the potential for spectral distortions as a probe of standard slow-roll inflation physics.

\subsection{Primordial non-Gaussianity}

Spectral distortion anisotropies also can be used to probe local-type primordial nonGaussianity at small scales [44-46, 131-137], an exciting direction that complements other cosmological probes and could shed light on multi-field inflation scenarios [138]. As discussed above, the dissipation of primordial acoustic modes on small scales generates a guaranteed contribution to the isotropic $\mu$-and $y$-distortions. Non-Gaussian (NG) couplings between short- and long-wavelength modes create inhomogeneities in the amplitude of the small-scale power, which in turn lead to anisotropic spectral distortions that correlate with tracers of the long-wavelength modes [44, 45].

Broadly speaking, most of the information about the non-Gaussianity generated by different early-Universe models can be captured by the Fourier transform of the 3and 4-point correlation functions, respectively, the primordial bispectrum and trispectrum. At large scales, these have been tightly constrained by the Planck collaboration analysis of the CMB temperature and polarization anisotropies (respectively $T$ and $E$ ) bispectrum and trispectrum [139]. In contrast, cross correlations of $T$ and $E$ with $\mu$-distortions anisotropies probe an interesting class of bispectra (e.g. [45, 131, 132]) and poly-spectra (e.g. [140]) that peak in squeezed configurations, with one of the momenta much smaller than the others. In this category falls the local model bispectrum, whose amplitude $f_{\mathrm{NL}}^{\mathrm{loc}}$ can discriminate between single and multi-field inflation. The measurement of $\mu-T$ cross correlation will set the first upper bound on $f_{\mathrm{NL}}^{\text {loc }}$ on small scales $\left(k \approx 740 \mathrm{Mpc}^{-1}\right)$, shedding light on possible scale-dependence of the NG parameters $[46,131]$, thus complementing parametric searches performed on the vastly different CMB anisotropy scales $[141,142]$.

Spectral distortion anisotropies can indeed be targeted by both CMB spectrometers or differential CMB imagers. The angular cross-correlation $\mu-T$ (see left panel of Fig. 4) can be simply expressed as [45, 134]

$$
C_{\ell}^{\mu T} \approx-12 f_{\mathrm{NL}}^{\mathrm{loc}}\langle\mu\rangle \int \mathrm{d} k \frac{2}{\pi} k^{2} \mathcal{T}_{\ell}^{T}(k) \frac{j_{\ell}\left(k r_{\mathrm{ls}}\right)}{5} P(k) \mathrm{e}^{-\frac{15 k^{2}}{8 k_{\mathrm{rec}}^{2}}},
$$

where $\langle\mu\rangle$ is the average dissipation $\mu$-distortion (defined in Eq. 1), $\mathcal{T}_{\ell}^{T}$ is the temperature transfer function, $r_{\mathrm{ls}}$ is the comoving distance to last scattering and $k_{\mathrm{rec}}$ is

\footnotetext{
${ }^{3}$ In practice we rescaled the Planck covariance matrix assuming factors of 2 error improvements for $A_{\mathrm{S}}$, $n_{\mathrm{S}}$ and $n_{\text {run }}$.
} 

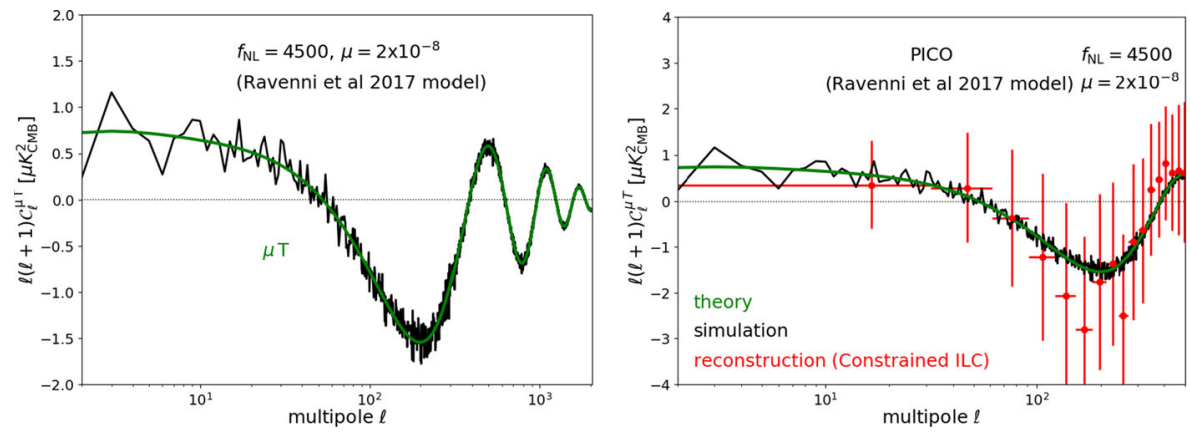

Fig. 4 Left: Expected $\mu$-T cross-power spectrum (2) between CMB temperature and $\mu$ distortion anisotropies for $\langle\mu\rangle=2 \times 10^{-8}$ and $f_{\mathrm{NL}}^{\text {loc }}\left(k \simeq 740 \mathrm{Mpc}^{-1}\right)=4500$. Right: Reconstructed $\mu-T$ correlation signal after foreground removal with the Constrained-ILC method for PICO. Figures from [143]

the diffusion-damping scale at the epoch of recombination. The exact degeneracy between $f_{\mathrm{NL}}^{\mathrm{loc}}$ and the spectral distortion monopole means that to interpret the data in principle measurable with a differential CMB imaging instrument - also requires an absolute measurement [134]. A larger monopole would enhance the signal, and thus render NG signals more observable. While we limit our discussion to the correlation with temperature anisotropies, further improvements in sensitivity to $f_{\mathrm{NL}}^{\text {loc }}$ can be achieved by considering $y-T, y-E$ and $\mu-E$ correlations [134-136].

Figure 4 (right) shows the reconstruction of the $\mu-T$ correlation signal between CMB temperature and $\mu$-distortion anisotropies for the $P I C O$ experiment [144] after foreground mitigation and deprojection of residual CMB temperature anisotropies in the reconstructed $\mu$-map with the Constrained-ILC method [145] (to eliminate spurious residual $T T$ correlations in the $\mu$ - $T$ cross-power spectrum). For a PICOtype space mission, using $f_{\mathrm{NL}}^{\mathrm{loc}}\left(k \simeq 740 \mathrm{Mpc}^{-1}\right)=4500$ and $\langle\mu\rangle=2 \times 10^{-8}$ as a fiducial values, the $\mu-T$ cross-power spectrum is recovered without bias at large angular scales and detected at $2 \sigma$ significance when including modes at $2 \leq \ell \leq 500$. This result is not biased by secondary and line-of-sight effects [137]. For $f_{\mathrm{NL}}^{\text {loc }}\left(k_{0}\right) \simeq$ 5 at CMB pivot scale, $k_{0}=0.05 \mathrm{Mpc}^{-1}$, this would impose a limit of $n_{\mathrm{NL}} \lesssim 1.6$ on the spectral index of $f_{\mathrm{NL}}^{\text {loc }}(k) \simeq f_{\mathrm{NL}}\left(k_{0}\right)\left(k / k_{0}\right)^{n_{\mathrm{NL}}-1}$ for scale-dependent nonGaussianity, providing a new way to constrain non-standard early-Universe models (e.g. multifield inflation). These limits complement those from Planck and future experiments like the SKA and SphereX, which could reach $f_{\mathrm{NL}}^{\text {loc }} \simeq \mathcal{O}(1)[146,147]$ at much larger scales.

As demonstrated in [143], coverage at frequencies below $40 \mathrm{GHz}$ is more important for a detection of the enhanced $\mu-T$ correlation than at high frequencies. This is because the $\mu$-distortion energy spectrum is more degenerate with the CMB temperature blackbody spectrum at high frequencies. Nevertheless, high frequencies are needed to clean dust foregrounds at large angular scales, necessitating broad spectral coverage $(v \simeq 20-800 \mathrm{GHz}$ ) for this science objective. In addition, it was shown in [143] that extended spectral coverage at frequencies $v \lesssim 40 \mathrm{GHz}$ and 
$v \gtrsim 400 \mathrm{GHz}$ provides more leverage for constraining the $\mu-T$ cross-power spectrum than increased channel sensitivity over a narrower spectral range. Finally, since most of the $\mu-T$ correlation is contained at large angular scales $2 \leq \ell \leq 500$ (Fig. 4; left), a space mission scanning the full sky with broad spectral coverage and moderate angular resolution $\left(\delta \theta \simeq 0.5^{\circ}\right)$ is highly motivated.

The magnitude of this constraint prima-facie is poor compared to the benchmark set by Planck, and, e.g., to the prospect of measuring NG signatures in the galaxy bias $[148,149]$. However, such a comparison implicitly assumes the lack of any scaledependence of the NG parameters. Albeit plausible, the absence of any running of $f_{\mathrm{NL}}^{\text {loc }}$ over more than 4 orders of magnitude is per se a valuable hint that can lead to further understanding of the underlying physics. Any measurement of this kind would also be a first step toward the invaluable goal of reaching cosmic-variance-limited determination of the $\mu-T$ cross correlation which is, to this day, the only proposed way to reach the lower bound set by the Maldacena consistency relation [150].

\subsection{CMB spectral distortions as a probe of dark matter and particle physics}

The search for dark matter is another example of how spectral distortions probe new physics. Non-baryonic matter constitutes $\simeq 25 \%$ of the energy density of the Universe, but its nature remains unknown. The long-favored WIMP-scenario is under increasing pressure [153-158], and emphasis is gradually shifting focus towards alternatives, prominent examples being axions, sterile neutrinos, sub-GeV DM or primordial black holes [159-165].

To solve this puzzle, a coordinated multi-tracer approach that combines different particle physics and cosmological probes is needed. Measurements of the CMB anisotropies themselves have clearly helped to establish the presence of DM on cosmological scales and provided tight constraints on DM annihilation and decay [166-173] and DM-SM-interactions [174-178]. However, for DM annihilation and decay CMB anisotropies quickly lose constraining power before recombination $(z \gtrsim$ $10^{3}$ ), being impeded by cosmic variance. Similarly, measurements of light-element abundances [151, 152, 166, 179], which are only sensitive to non-thermal energy release above nuclear dissociation thresholds in the pre-recombination era $[17,180]$, mostly saturated their limits due to astrophysical uncertainties. This is where $C M B$ spectral distortions offer a valuable complementary probe. For decaying particle scenarios, distortions are sensitive to particles with lifetimes $t_{X} \simeq 10^{6}-10^{12} \mathrm{~s}[13,17$, 181-186], providing direct measurement of particle lifetimes via $r$-type distortions $[16,17]$. Existing limits from light-element abundances on the particle yield variable, which provides a measure of the relic abundance and mass of the particle (e.g., see [151], for details), could thus be improved by orders of magnitude (see Fig. 5), reaching deep into the primordial Universe for large energy injection [187]. Similarly, annihilating particles can be constrained using distortions: the $\mu$-distortion is sensitive to light particles ( $m \lesssim 100 \mathrm{keV}$ ) and complements $\gamma$-ray searches for heavier particles, being sensitive to s- and p-wave annihilation $[16,188]$. The rich spectral information added by various non-thermal processes [18, 22-24, 26, 27] will allow us to glean even more information about the nature of dark matter, placing limits on the importance of different decay or annihilation channels. 


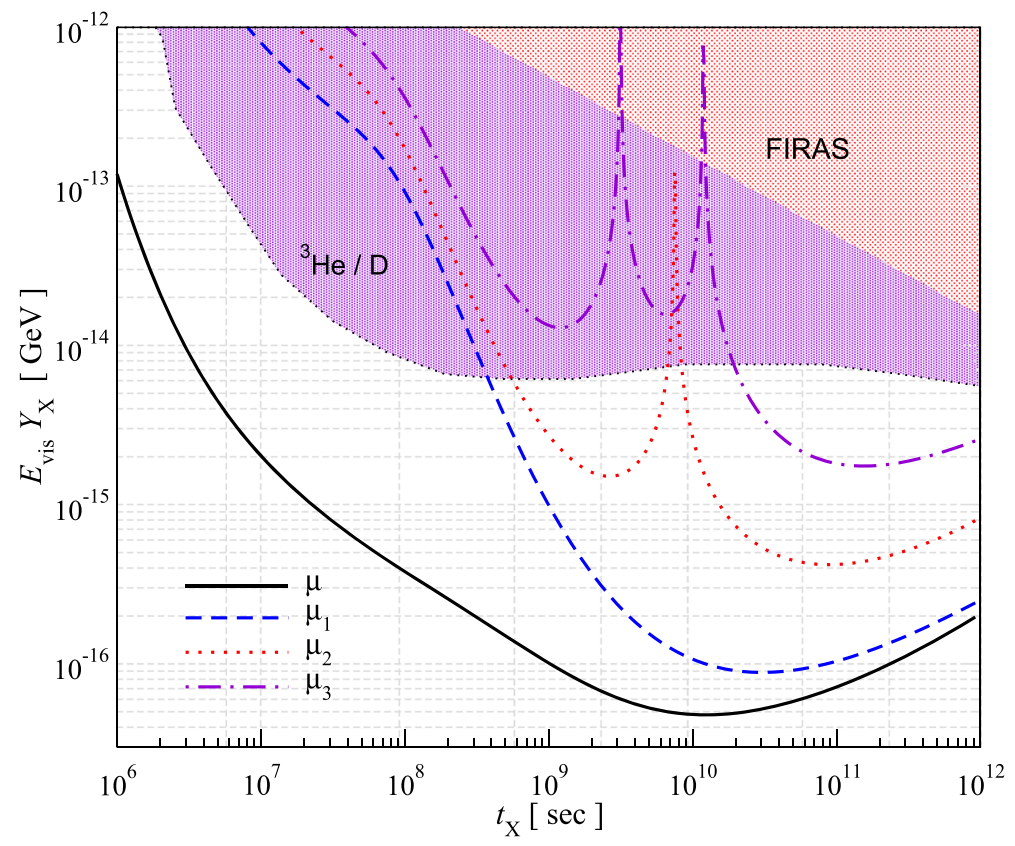

Fig. 5 Constraints on the yield variable, $E_{\mathrm{vis}} Y_{X}$ (e.g., see [151], for details), from electromagnetic particle decay for varying lifetime, $t_{X}$. For the distortion forecast a spectral sensitivity of $\sigma(\mu) \simeq 10^{-8}$ (aka SuperPIXIE) was assumed. The parameters $\mu_{i}$ describe extra time-dependent information available from the $r$-type distortion (see [17], for details). For comparison we quote the constraints from [151, 152] for decays into $e^{+} e^{-}$derived from the ${ }^{3} \mathrm{He} / \mathrm{D}$ abundance ratio. Future spectral distortion measurements could improve the constraint on decaying particles with lifetimes $t_{X} \simeq 10^{7}-10^{12}$ by orders of magnitude. Using the $r$-type distortion we could furthermore break the degeneracy between particle yield and lifetime, should a significant distortion signal be detected [13, 17]. Figure adapted from [17]

More work is required, although it is already clear that in addition to the aforementioned examples distortions can meaningfully probe scenarios involving axions [189-191], gravitino decays [182, 192], cosmic strings [193, 194], DM-SMinteractions [120, 172, 195], macros [196] and primordial magnetic fields [197-200]. This opens a path for studying a wide range of new physics.

\subsection{Primordial black holes}

CMB spectral distortions can also place stringent limits on the abundance of primordial black holes (PBHs) (e.g., [164, 201-203]). There is good motivation to study these scenarios, because PBHs with masses of $m_{\mathrm{PBH}} \simeq \mathcal{O}(10) M_{\odot}$ may explain the gravitational wave signals [204-206] emitted in the merger events of (primordial) binary black holes reported by LIGO / Virgo [207]. PBHs with masses in the range $m_{\mathrm{PBH}} \simeq 10^{-17} M_{\odot}-10^{-11} M_{\odot}[164,208]$ can furthermore still constitute $\simeq 100 \%$ of cold dark matter (see also [113, 202, 209]). Lastly, PBHs with masses $m_{\mathrm{PBH}} \simeq 3 \times 10^{3} M_{\odot}-10^{5} M_{\odot}$ may form the seeds for supermassive black holes 
(SMBHs) that grow to their current sizes merely by continuous (sub-)Eddington accretion, solving a long-standing problem in cosmology [210-212].

To produce $\mathrm{PBHs}$, we expect a large primordial curvature perturbation $P_{\zeta} \gtrsim 0.03$ with a critical density perturbation $\left.\delta_{c} \simeq 0.3-0.4[213,214]\right)$ at small scales to have collapsed directly into a black hole during the radiation-dominated Universe (see also [215] for the case of an early matter-dominated Universe). It is known that large curvature perturbations can indeed be produced by some classes of inflation [101, 202, 216, 217], curvaton [218, 219] and preheating scenarios [220], providing further motivation to study these cases.

With this picture in mind, various ways to limit the abundance of PBHs have been proposed in cosmology and astrophysics (e.g., see [164, 221], and references therein). Among these methods, the limits from observations of CMB in terms of spectral distortions and modifications to the recombination history of atoms are most robust. Here, we focus on the former effects (see also references about the latter effects from evaporations of PBHs [164, 173, 222] and accretions onto PBHs [223, 224]). There are two types of constraints on PBHs from the spectral distortions of CMB, each of which is individually induced by 1) dissipation of a large density perturbation which is expected to collapse into PBHs and 2) electromagnetic particles emitted by evaporating PBHs. For case 1), it is notable that this effect occurs whenever PBHs are formed by a Gaussian fluctuations. The current bound on the spectral distortions has already excluded PBH masses of $m_{\mathrm{PBH}} \gtrsim 3 \times 10^{4} M_{\odot}$ [211]. In addition, from limits set by Big Bang Nucleosynthesis (BBN) in order not to dilute baryon due to the dissipation [225, 226], we can exclude masses in the range $3 \times 10^{3} M_{\odot} \lesssim m_{\mathrm{PBH}} \lesssim$ $3 \times 10^{4} M_{\odot}$ [227]. Overall, PBHs with masses $m_{\mathrm{PBH}} \gtrsim 3 \times 10^{3} M_{\odot}$ can thus not make more than a fraction $f_{\mathrm{dm}} \simeq 10^{-8}$ of the DM for Gaussian density perturbation. This already puts PBHs as seeds of SMBHs under strong pressure, and future spectral distortion experiments could further tighten these limits. However, if large non-Gaussian curvature perturbation were created at the relevant small scales (not probed by CMB anisotropies), the above bound could become much milder [228]. In this case, PBH clusters would be expected (e.g., [229]), which could furthermore lead to anisotropic distortion signals. Future measurements of CMB spectral distortions could shed further light on these scenarios.

On the other hand, for case 2), existing limits from CMB spectral distortion [230] are currently $\simeq 10^{3}$ times weaker than those from BBN (see Fig. 6 of [164]), which tightly constrain masses in the range $10^{9} \mathrm{~g} \lesssim m_{\mathrm{PBH}} \lesssim 3 \times 10^{13} \mathrm{~g}$. However, in the future, the $\mu$-distortion constraints could be improved beyond the BBN limits, probing masses in the range $10^{10} \mathrm{~g} \lesssim m_{\mathrm{PBH}} \lesssim 3 \times 10^{12} \mathrm{~g}$.

We close by mentioning that $\mathrm{CMB}$ spectral distortions are also indirectly sensitive to $\mathrm{PBHs}$ with masses $m_{\mathrm{PBH}} \simeq \mathcal{O}(10) M_{\odot}[202,228]$. Perturbations forming PBHs with $m_{\mathrm{PBH}} \simeq \mathcal{O}(10) M_{\odot}$ correspond to wavenumbers $k \simeq 10^{6} \mathrm{Mpc}^{-1}$, which is well outside the range $1 \mathrm{Mpc}^{-1} \lesssim k \lesssim 10^{4} \mathrm{Mpc}^{-1}$ to which dissipation spectral distortions are directly sensitive. However, assuming a large enhancement of the primordial power spectrum at $k \simeq 10^{6} \mathrm{Mpc}^{-1}$ also means that the perturbations at $1 \mathrm{Mpc}^{-1} \lesssim k \lesssim 10^{6} \mathrm{Mpc}^{-1}$ ought to be modified. Depending on the mechanism creating the large perturbation at $k \simeq 10^{6} \mathrm{Mpc}^{-1}$, the transition from the low power 
at large angular scales is more or less rapid and thus can be probed using future CMB spectral distortion measurements [88].

\subsection{Axion-like particles}

Axions or Axion-Like Particles (ALPs) are predicted in multiple particle physics scenarios [231-236], and their discovery would mark a paradigm shift in the framework of the standard models of cosmology and particle physics. Several particle physics experiments [237] such as CAST [238], ALPS-II [239], MADMAX [240], ADMX [241], CASPER [242] are looking for the signatures of axions or ALPs over a wide range of masses. Along with the particle physics experiments, cosmological probes such as CMB anisotropies and large-scale structure are exploring the gravitational effects of ALPs on the matter density with the potential to discover ALPs if it constitutes dark matter [165, 243-247]. The other possibility to probe ALPs (even if they are a fraction of DM) is by studying their coupling with photons in the presence of an external magnetic field [248-250].

The coupling between ALPs and photons $g_{\gamma \gamma a}$ leads to oscillations between photons and ALPs and vice versa in the presence of an external magnetic field. This effect is one of the cleanest windows for detecting ALPs. The signatures of this nongravitational interaction of ALPs with photons distort their energy spectrum and thus can be detected robustly if the energy spectrum of the source is well-known. The radiation field of CMB provides us with an excellent source which can be used to detect the distortions due to ALPs [191]. The ALPs distortion ( $\alpha$-distortion) is imprinted on the CMB while it is passing through the external magnetic field of the intergalactic medium (IGM), inter-cluster medium, voids and Milky Way. The conversion from photons to ALPs can be classified into two types, namely the resonant conversion and the non-resonant conversion.

The resonant conversion of CMB photons into ALPs takes place when the photon mass in the plasma equals the mass of ALP. The polarization state of the CMB photon which is parallel to the external magnetic field gets converted into ALPs depending upon the strength of the magnetic field. As a result, it leads to a polarized spectral distortion of the CMB blackbody with a unique spectral shape. Also due to inhomogeneities in the magnetic field of the astrophysical systems, the observed polarized distortion varies spatially which leads to a unique spatial structure that differs from any other known spectral distortions and foreground contaminations. Though the resonant conversion of CMB photons can take place in different kinds of astrophysical systems it can be best measured in Milky Way and galaxy clusters.

The Milky Way's galactic magnetic field induces a large angular scale spectral distortion as shown in Fig. 6. This signal can be targeted with a low-resolution spectrometer like PIXIE or SuperPIXIE. While polarization information increases the sensitivity, even intensity distortion measurements can be used to derive stringent constraints. The shape of the ALPs distortion depends upon the mass of the axions and the density of electrons in the Milky Way. For the best-fit model of electron density [251] and magnetic field [252, 253] of the Milky Way, ALPs in the mass range from a $m_{\mathrm{ALP}} \simeq$ few $\times 10^{-13} \mathrm{eV}$ to a few $\times 10^{-12} \mathrm{eV}$ can be probed by the process of resonant conversion. The measurement of this large angular scale spectral 


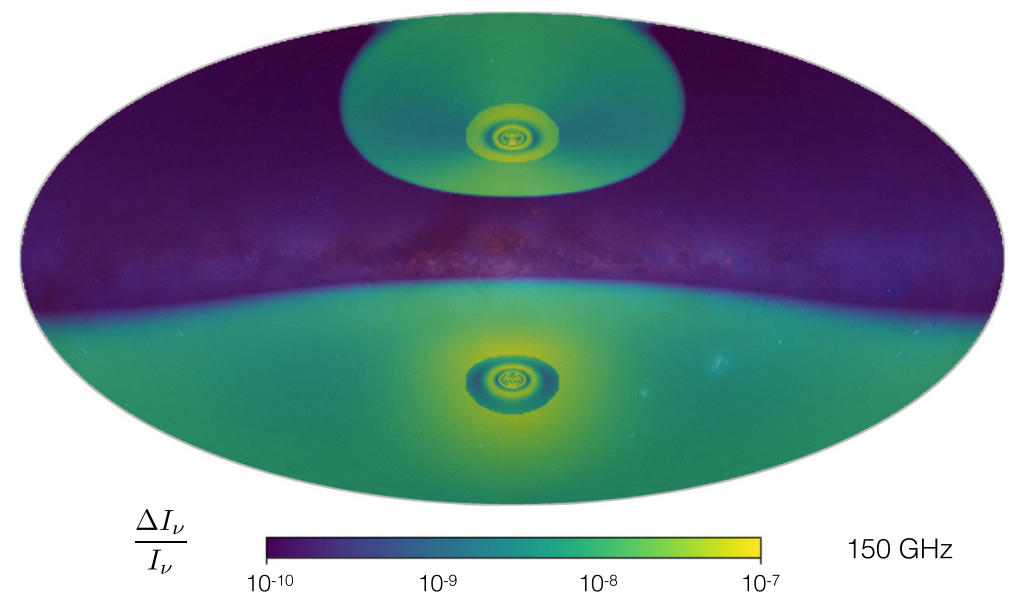

Fig. 6 The resonant conversion between CMB photons into ALPs in the presence of galactic magnetic field produces spatially varying spectral distortion. We plot the expected spectral distortion signal $\Delta I_{v} / I_{v}$ at $150 \mathrm{GHz}$ for ALP of mass $m_{a}=5 \times 10^{-13} \mathrm{eV}$ and photon-axion coupling $g_{\gamma \gamma a}=10^{-11} \mathrm{GeV}^{-1}$ using the best-fit model of galactic electron density [251] and magnetic field [252, 253]. The intensity and the shape of the distortion varies with the mass of ALPs $m_{a}$ and the coupling $g_{\gamma \gamma a}$ and is most pronounced at large angular scales. An optical image of our galaxy was overlaid for reference. The northsouth asymmetry of the signal stems from the structure of the galactic magnetic field and electron density

distortion signal requires both wide frequency- and sky-coverage, which is possible only with space-based CMB missions. The same physical effect also arises in galaxy clusters [254] and produces polarized spectral distortions that can be measured using high-resolution CMB experiments with an imaging telescope [59, 255].

Along with the resonant conversion of CMB photons into ALPs, there will also be a non-resonant conversion of CMB photons into ALPs, as the CMB photons propagate through the turbulent magnetic field of our galaxy, IGM and voids [191]. This leads to an unpolarized spectral distortion of the CMB blackbody. This avenue will provide stringent constraints on the coupling strength $g_{\gamma \gamma a}$ for all the masses of ALPs below $\simeq 10^{-11} \mathrm{eV}$. The first constraint of this kind of distortion is obtained from the data of the Planck satellite [256].

This new probe of ALP physics will be accessible with CMB spectrometers like PIXIE or SuperPIXIE. In this way, we can explore a new parameter space of the coupling strength $g_{\gamma \gamma a}$ and ALP masses, which are currently beyond the reach of particle-physics experiments. Spectral distortions are capable of discovering ALPs even if they are a fraction of DM and hence will open a completely new complementary window for studying ALPs in nature. The discovery space is enormous and provides a direct cosmological probe into the string axiverse [235].

\subsection{The cosmological recombination radiation}

The recombination process causes another small but inevitable distortion of the CMB. Line emission from hydrogen and helium injects photons into the CMB, which after redshifting from $z \simeq 10^{3}$ are visible today as complex frequency structure in 
the microwave bands (Fig. 7) [19, 257-264]. The cosmological recombination radiation (CRR) has a simple dependence on cosmological parameters and the dynamics of recombination; since it includes not only hydrogen but also two helium recombinations, it probes eras well beyond the last-scattering surface observed by CMB anisotropies [20, 21, 265]. Modern computations now include the bound-bound and free-bound contributions from hydrogen, neutral helium and hydrogenic helium and thus allow precise modeling of the total signal and its parameter dependences [21].

Cosmological recombination process is crucial for understanding and interpreting the CMB temperature and polarization anisotropies [266-269]. It is thus critical to test our physical assumptions during this era [270-273]. The CRR provides one of the most direct methods to achieve this. It should enable a pristine measurement of the primordial helium abundance, long before the first stars have formed. On the other hand, it is fairly insensitive to the effective number of neutrino species and thus can help breaking the degeneracy with the primordial helium abundance [21].

The CRR is also directly sensitive to new physics affecting the recombination dynamics. Decaying or annihilating particles could enhance the total emission caused by the primordial atoms [274], leaving features that may help determining the time-dependence of the process through uncompensated atomic transitions in the pre-recombination era (e.g., (e.g., [23]). The contributions from both helium recombinations furthermore arise from significantly earlier phases (redshifts $z \simeq 2000$ and 6000 , respectively; cf., Fig. 1). This opens a new window to the primordial Universe that cannot be directly accessed in another way. Measurements of the CRR will also allow us to directly map the baryon density and other cosmological parameters at

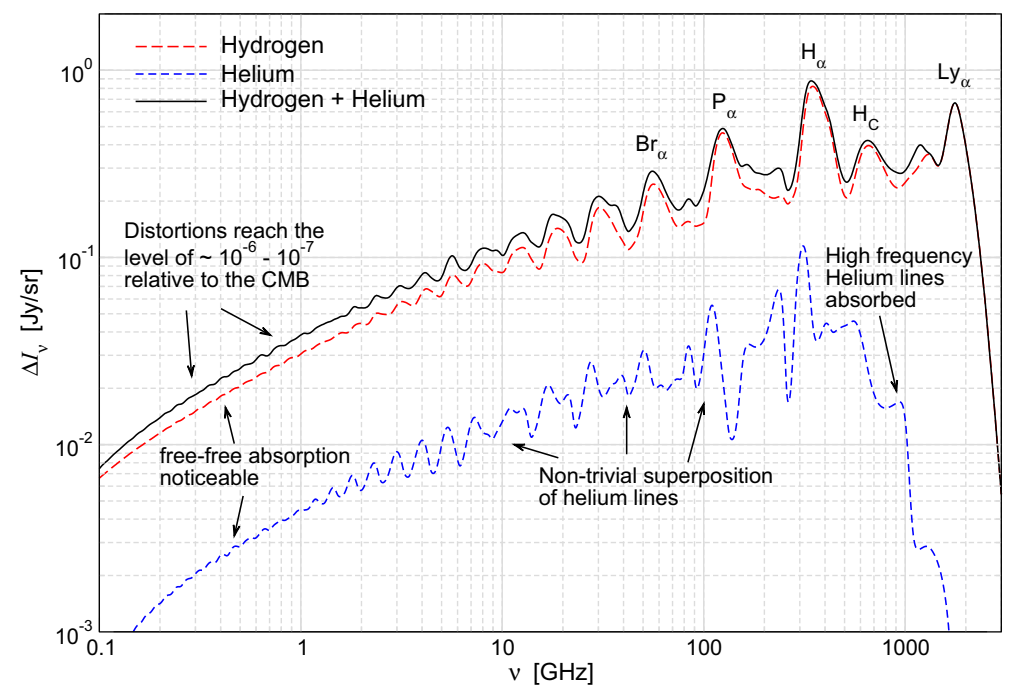

Fig. 7 The cosmological recombination radiation arising from the hydrogen and helium components. The helium distortion (blue) is the net accumulation of the HeI and HeII emission along with other effects (i.e. helium absorption and collisions). The spectral series of hydrogen have also been highlighted. The unique spectral shapes given by the CRR would provide us with a revolutionary new cosmological probe of the atomic physics in the early Universe 
$z \simeq 10^{3}$ across the sky, providing an independent test of statistical isotropy [21]. And finally, variations of fundamental constants should leave observable imprints in the shape of the CRR at otherwise inaccessible epochs [21].

Although the CRR is one of the smallest signals expected in standard $\Lambda$ CDM (see Fig. 9), its detection is on par with the larger $\mu$-distortion from acoustic damping (see Section 4.2). This is because the CRR and its derivatives with respect to the cosmological parameters have many spectral features (e.g., Fig. 7 here and also Fig. 5 and 6 of [21]), making it easier to distinguish the signals from the much brighter but smoother foregrounds [275, 276]. For this reason, at very low frequencies $(v \simeq$ few $\times \mathrm{GHz}$ ) the CRR could in principle be targeted from the ground using concepts like APSERa [276], however, to overcome atmospheric noise and access the more structured signal at high frequencies $(v \gtrsim 100 \mathrm{GHz})$ a space mission will be required [275]. SuperPIXIE could detect the distortion at the level of $\simeq 2 \sigma$ (Section 4.2), opening a way to directly test our physical understanding of the $z=10^{3}$ Universe. A more detailed parameter forecast using the CRR and the related observational challenges including distortion foregrounds is given in [277].

\subsection{Reionization and structure formation}

The epoch of reionization and the formation of cosmic structures mark additional important transitions in the evolution of our Universe. The largest all-sky spectral distortion signal is indeed caused by the reionization and structure-formation processes [52, 278-282]. Energy output from the first stars, accreting black holes, and gravitational shocks heats the baryons and electrons, which then up-scatter CMB photons to create a $y$-type distortion. The overall distortion is expected to reach $y \simeq$ few $\times 10^{-6}$ [52, 281, 283, 284], only one order of magnitude below the upper bound placed by $C O B E /$ FIRAS. Such a distortion must exist and provides a measure of the total thermal energy of (ionized) baryons in the Universe. Spectrometers like PIXIE or SuperPIXIE will measure this distortion to sub-percent precision (Section 4.2, Fig. 10). The low-redshift $y$-distortions from reionization and structure formation are furthermore anisotropic (e.g., [52-54, 56]) and thus open new opportunities for cross-correlation studies (e.g., with CMB and $21 \mathrm{~cm}$ tomography).

A large part of the low-redshift Compton- $y$ signal is due to halos with masses $M \simeq 10^{13} M_{\odot}$, which contain virialized gas with an electron temperature of $k T_{\mathrm{e}} \simeq$ $2-3 \mathrm{keV}$. This causes a relativistic temperature correction (rSZ) [287-292] to the $y$-distortion that can directly tell us about feedback mechanisms [284]. In addition, both $y$ and the rSZ distortion depend directly on the shape and amplitude of the halo mass function, providing another cosmological measure of the growth of structure. The level of the relativistic contributions is, however, uncertain and current estimates based in X-ray temperature-mass relations may underestimate the signal by a factor of $\simeq 1.5-2[293,294]$, thus further increasing the detectability of this signal. Nevertheless, with spectrometers like PIXIE or SuperPIXIE, the average relativistic temperature could be determined to tens of standard deviations (Section 4.2).

With simultaneous high-precision measurements of both $y$ and rSZ, we will be able to place tight constraints on models of feedback in galaxy formation. This is illustrated in Fig. 8, which shows the range of current predictions for these quantities 


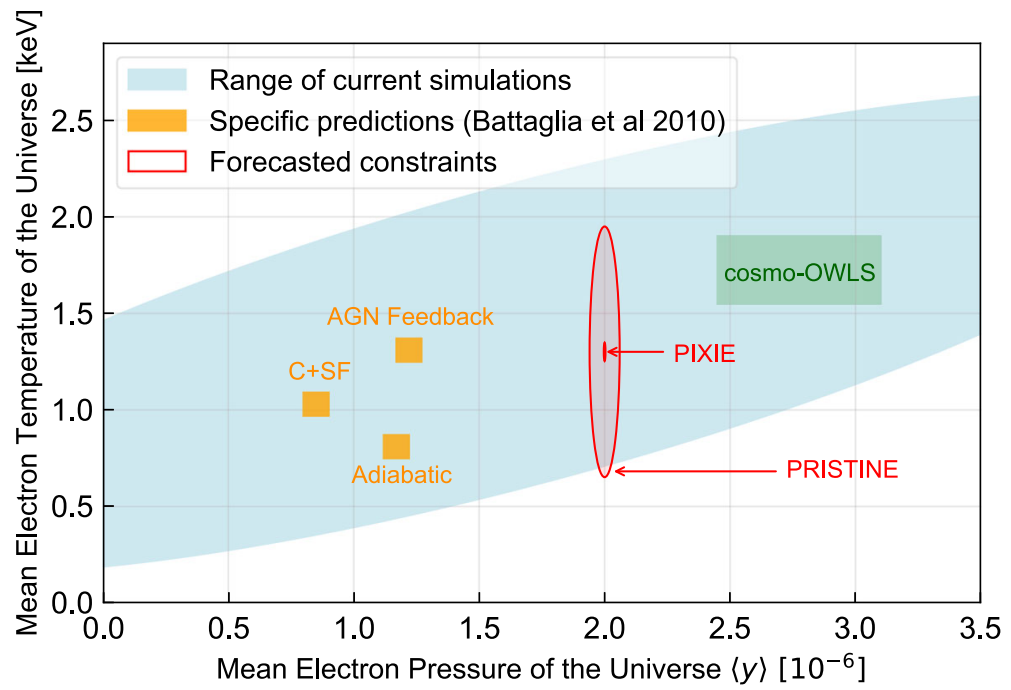

Fig. 8 Theoretical predictions and forecasted constraints for the late-time Compton- $y$ and relativistic SZ spectral distortions due to structure formation and reionization, with $y=2 \times 10^{-6}$ and $k \mathrm{~T}_{\mathrm{e}}=1.3 \mathrm{keV}$ as fiducial values [284]. The light blue ellipse encompasses the approximate range of several current predictions for these quantities. Each of the labeled squares denotes a specific prediction from the simulations of [285], where only the sub-grid feedback model is varied. The green rectangle indicates the range of results for the cosmo-OWLS simulations [286]. The red ellipses show the forecasted constraints on these quantities for PRISTINE and PIXIE (is hardly visible for PIXIE); more powerful missions in the Voyage 2050 program would provide even tighter constraints

from state-of-the-art cosmological hydrodynamics simulations [285, 286], including precise predictions from different feedback implementations. The figure also shows forecasted constraints for PRISTINE and PIXIE, as illustrative spectral distortion missions. It is clear that such measurements will strongly distinguish between current sub-grid feedback models, yielding significant breakthroughs in our understanding of galaxy formation. A direct measurement of the average rSZ temperature would also shed new light on the missing baryon problem [280] without the need to resolve the warm-hot-intergalactic medium, a unique opportunity that we should make use of in the future.

The late-time $y$-distortion has an additional contribution at the level of $y \simeq$ few $\times 10^{-8}$ due to second-order Doppler terms from the large-scale velocity field $[279,295]$. This signal and the average distortion from the reionized $\simeq 10^{4} \mathrm{~K}$ gas could be accessed by masking resolved SZ clusters, or by isolating the latter signal through cross-correlations with galaxy and cluster catalogs. This procedure also reduces one of the largest primordial distortion foregrounds, the low-redshift $y$ distortion itself, and would therefore allow us to tighten the upper limits on early energy release occurring at $z \simeq 10^{3}-10^{4}$, a unique opportunity for combining CMB spectroscopy and imaging. Measurements at $v \gtrsim 500 \mathrm{GHz}$ will furthermore probe 
the total cosmic ray energy density of the Universe through the non-thermal relativistic SZ effect [25, 296-298]. And finally, at low frequencies, the free-free distortion from the ionized gas will become visible, probing the thermal state and the clumping of the intergalactic medium [299-303], as well as time-dependent effects in the pre-recombination era [13].

\subsection{Line intensity mapping}

The measurement of the integrated Far-IR background [304] was a significant legacy of the COBE/FIRAS mission. The amplitude of the Far-IR background suggests that half of the starlight in the Universe is absorbed and reprocessed through thermal dust emission. Similarly to the other spectral distortions, the extragalactic background light provides a synoptic view of energetic processes in all galaxies. The $C O B E / F I R A S$ measurement of integrated dust emission became a reference point for two decades of fruitful observations to resolve the sources of this emission into individual galaxies. The continuum radiation spectrum has no identifiers for the redshift of its emission, but cross-correlation with a galaxy redshift survey permits some dissection of the emission into its constituent redshifts [305]. Future spectral surveys will be able to measure not only the dust continuum but also the integral of diffuse line radiation (namely $\mathrm{CO}$ ladder, [CII] and [NII]), which maps directly to redshift. This approach of Line Intensity Mapping has attracted significant attention in recent years as a probe for both galaxy evolution and fundamental cosmology [306, 307]. Line emission traces cold, molecular gas (a precursor to star formation) and line emission excited by star formation [308]. COBE/FIRAS has insufficient sensitivity to extract this emission, and searches in the Planck data have hit fundamental limits $[309,310]$ at a $3 \sigma$ excess consistent with [CII] emission. New instruments are needed to constrain this signal [311].

A space-borne FTS is a unique platform for intensity mapping. It provides 1) access to the monopole of line emission [312, 313], 2) access to the largest modes of anisotropy on the sky, and 3) a highly precise passband calibration through differencing with a blackbody reference. Cross-correlation with a galaxy redshift survey allows the line signal to be extracted unambiguously from uncorrelated Milky Way foregrounds and may ultimately mitigate cosmic variance [314]. This crosscorrelation measures not only the line brightness, but also the SED of average galaxies as a function of frequency and time. PIXIE and SuperPIXIE will have a sufficient number of spectral channels to separate the correlated line and continuum emission [315]. In each of its frequency channels, PIXIE is expected to make a high significance detection of [CII] emission, from the present to $z \simeq 2$ [314], and detection of the CO $J$ ladder at $z<1$ (depending on poorly constrained emission models). Access to large volumes also permits probes of fundamental physics through searches for primordial non-Gaussianity. The authors of [316] find that a 4-year PIXIE survey could

constrain $\sigma\left(f_{\mathrm{NL}}^{\text {loc }}\right)=2.1$, which is comparable to future goals of SKA and SphereX $[146,147]$ and complements limits obtained with $\mu$-anisotropies (Section 3.2). With $10 \times$ the capability, SuperPIXIE should reveal redshifted line emission monopole and 
anisotropy in all frequency channels. Much like for $C O B E$ /FIRAS, observations of integrated, redshifted line emission will provide a complement to efforts to catalog line emission from individual sources [317].

\subsection{Resonant scattering signals}

Interactions of $\mathrm{CMB}$ photons with atoms can imprint additional frequency-dependent signals through resonant line [318-325] and Rayleigh scattering effects [326, 327], or via collisional emission processes [325, 328-330], providing independent ways of learning about recombination, the dark ages and reionization. A detection of these frequency-dependent signals, even at large angular scales, is generally limited by sensitivity, foregrounds and especially by the accuracy of the inter-calibration between channels. The required level of precision will be naturally achieved by the proposed mission concepts for spectral distortions discussed here.

Importantly, some of the signals can be detected using a spectrometer with moderate angular resolution $(\ell \lesssim 300)$. For example, the resonant scattering of CMB photons in the $\mathrm{H} \alpha$ line during cosmological recombination [321, 324] is detectable with PIXIE or SuperPIXIE, providing a crucial demonstration of the methodology, which can be used for other lines (e.g. $\mathrm{P} \alpha$ ) and novel polarization signals (i.e., $T E$ and $E E$ from $\mathrm{H} \alpha$ ) with a PRISM-like mission that hosts both a CMB spectrometer and high-resolution imager [31]. The resonant scattering of CMB photons by the finestructure lines of metals and heavy ions (i.e., OI, OIII, NII, NIII, CI, CII) produced by the first stars can also be observed at angular scales around the first Doppler peak. This effect causes a blurring of the original CMB anisotropy field on intermediate angular scales given by $\delta C_{\ell} \simeq-2 \tau_{\mathrm{X}} C_{\ell}^{\mathrm{CMB}}[320,323]$, where $\tau_{\mathrm{X}}$ denotes the optical depth associated to a given transition $\mathrm{X}$ and $C_{\ell}^{\mathrm{CMB}}$ is the primordial CMB anisotropy angular power spectrum generated at $z \simeq 1100$. Typical frequencies involved in a few of the most relevant lines are $v_{\mathrm{obs}} \simeq 190 \times 10 /(1+z) \mathrm{GHz}, 475 \times 10 /(1+z) \mathrm{GHz}$, and $206 \times 10 /(1+z) \mathrm{GHz}$ for the [CII] $157.7 \mu \mathrm{m}$, [OI] $63 \mu \mathrm{m}$, and [OI] $145 \mu \mathrm{m}$ transitions, respectively. Here, $z$ denotes the resonant scattering redshift. An FTS can provide relative calibration between different frequency channels at the level of few nK for $v<600 \mathrm{GHz}$, thus enabling a sensitivity to values of $\tau_{\mathrm{X}}$ as low as $\simeq 10^{-5}$. As shown in Fig. 8 of [59], this level of inter-channel calibration uncertainty can shed light on the history of the metal pollution of the IGM during the end of the dark ages and the reionization epoch, thus constituting an alternative window to those cosmological times that is totally complementary to $\mathrm{HI} 21 \mathrm{~cm}$ observations.

Finally, the UV radiation field generated by stars at the end of the Dark Ages and during the reionization epoch influences the spin temperature associated with fine-structure transitions like [OI] $63 \mu \mathrm{m}$, [OI] $145 \mu \mathrm{m}$, and [CII] $157.7 \mu \mathrm{m}$ [329]. Through the Field-Wouthuysen effect, these transitions may be seen in absorption/emission against the CMB backlight, and thus will generate another type of distortion to the CMB blackbody spectrum at the $10^{-10^{-}}-10^{-7}$ level that is only reachable with a spectrometer in the ESA Voyage 2050 program. 


\section{The path forward with CMB spectral distortions}

\subsection{Technological challenges}

The seminal measurements of the CMB blackbody spectrum by $C O B E / F I R A S$ in the early '90s cemented the Hot Big Bang model by ruling out any energy release greater than $\Delta \rho_{\gamma} / \rho_{\gamma} \simeq 6 \times 10^{-5}$ (95\% c.l.) of the energy in CMB photons [1, 2, 6]. Advances since then, in both detector technology and cryogenics, could improve this constraint by four orders of magnitude or more (e.g., with experimental concepts like PIXIE [40, 41], PRISM [31], PRISTINE [331] or SuperPIXIE [58]), opening an enormous discovery space for both predicted distortion signals and those caused by new physics. On the timescales relevant to the Voyage 2050 program we expect to go beyond, surpassing the crucial threshold of detecting the dissipation $\mu$-distortion at more than $3 \sigma$.

$C O B E$ /FIRAS was not background limited; its sensitivity was instead set by phonon noise from the $1.4 \mathrm{~K}$ detector. Modern detectors, operating at $\simeq 0.1 \mathrm{~K}$, would have detector (dark) noise well below the intrinsic limit set by photon arrival statistics. The sensitivity of a background-limited instrument could be further improved by increasing its throughput or the integration time and, in a less trivial way, by modifying the mirror stroke (i.e., frequency-sampling) and reducing the optical load at high frequencies [58]. Combining replicas of the same telescope design can additionally enhance its capabilities. Modern blackbody calibrators now also reach sufficient thermal and spectral stability for the task. All these technological challenges can be overcome and it is possible to reach the required spectral sensitivities and coverage using FTS approaches that build on the legacy of COBE/FIRAS [31, 40, 41, 58, 331].

As a point of comparison, it is worth noting that the raw instrumental sensitivities under consideration for these spectral distortion measurements are in the same range as recently proposed CMB imaging missions for the 2030s. For example, the NASAproposed Probe of Inflation and Cosmic Origins (PICO) aims to achieve an overall map-level sensitivity of $\approx 0.5-1 \mu \mathrm{K}$-arcmin, after combining all of its 21 frequency channels (considering noise only, i.e., no foregrounds) [144]. Averaging over the full sky, this corresponds to a monopole sensitivity of $\approx 0.02-0.04 \mathrm{Jy} / \mathrm{sr}$ at a reference frequency of $150 \mathrm{GHz}$. This is even below the range considered for the ESA Voyage 2050 spectral distortion mission concepts shown in Fig. 9. Of course, more channels and absolute calibration are needed for the spectral distortion measurements in comparison to an imager, but nevertheless it is clear that the relevant raw sensitivities are entirely feasible [31, 40, 41, 58, 331].

The designs of previously considered spectrometer concepts have evolved significantly in the past few years due to our improved understanding of the foreground challenge (Section 4.2). PIXIE was proposed as a NASA mid-Ex mission (duration 4 years, resolution $\delta \theta \simeq 1.5^{\circ}$ ), while PRISTINE was put forward as an F-class mission to ESA (duration 2 years, resolution $\delta \theta \simeq 0.75^{\circ}$ ). The SuperPIXIE concept was described for the recent NASA Decadal Survey 2020 (duration 4-10 years, resolution $\delta \theta \simeq 0.5^{\circ}-2^{\circ}$ depending on the band) [58]. All these concepts used polarization-sensitive, absolutely-calibrated FTSs, with hundreds of spectral channels covering $v \simeq 10 \mathrm{GHz}-$ few $\times \mathrm{THz}$. The estimated sensitivity curves and channel 


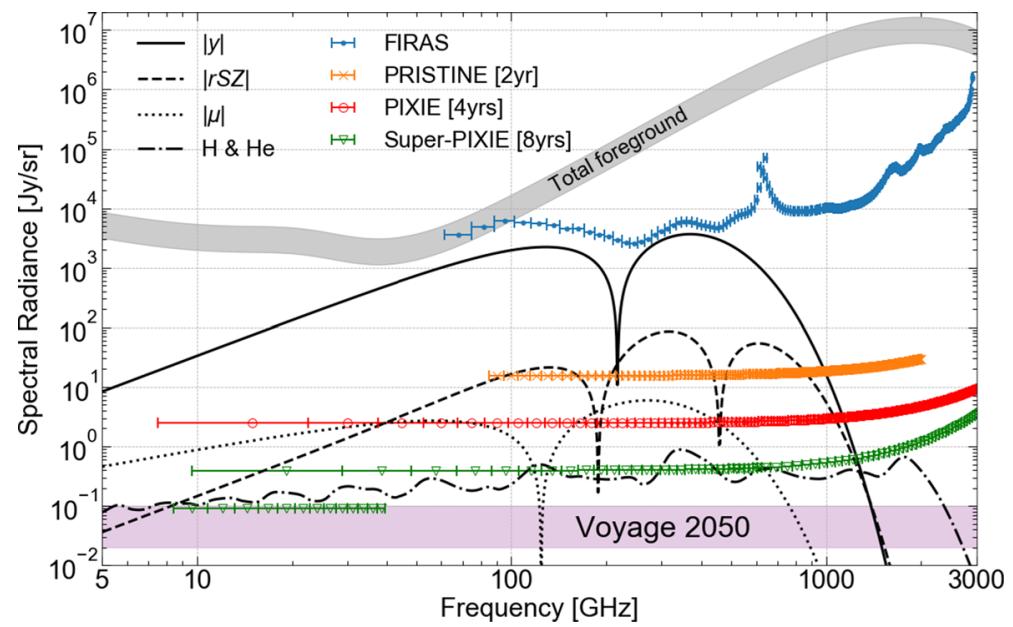

Fig. 9 Level of the expected CSM spectral distortion signals and cumulative astrophysical foregrounds. The estimated sensitivities for various mission concepts are illustrated with their channel distribution. The SuperPIXIE high and mid-frequency bands merge around $v \simeq 600 \mathrm{GHz}$. Within the ESA Voyage 2050 program the $\simeq 0.01-0.1 \mathrm{Jy} / \mathrm{sr}$ sensitivity level could be targeted, yielding clear detections of $\mu \simeq 2 \times 10^{-8}$ and also the CRR

distributions are illustrated in Fig. 9 together with the foreground and spectral signals. To improve foreground mitigation, for SuperPIXIE the frequency range was split into three separate bands (each with many channels). The anticipated spectral distortion constraints are summarized in Fig. 10. SuperPIXIE can reach $\sigma(y) \simeq 1.6 \times 10^{-9}$, $\sigma\left(k \mathrm{~T}_{\mathrm{e}}\right) \simeq 0.02 \mathrm{keV}, \sigma(\mu) \simeq 7.7 \times 10^{-9}$ and $\sigma(\Delta T)=10 \mathrm{nK}$ in eight years of

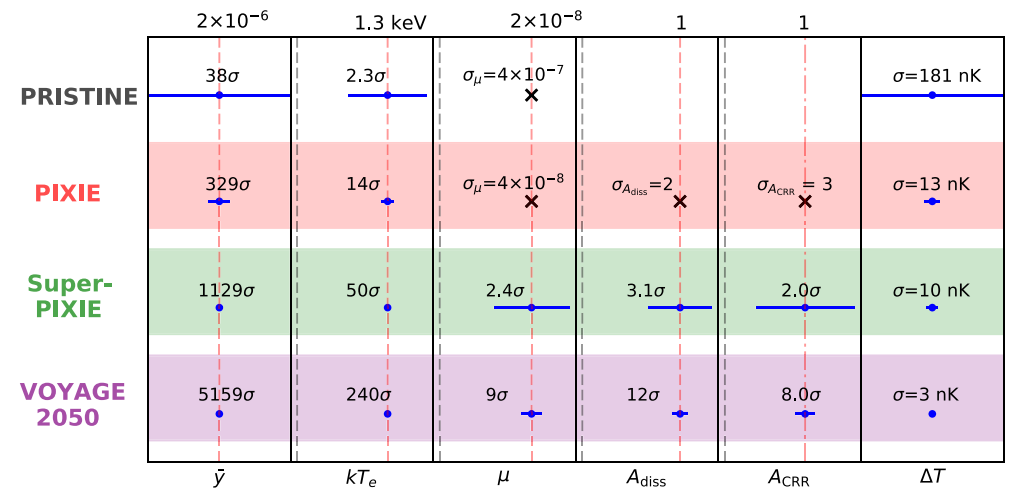

Fig. 10 Anticipated distortion signal error estimates after foreground marginalization. Each panel depicts the evolution of measurement errors with improved sensitivity of the proposed experiments. The red dashed line marks the fiducial values for the parameters, while the black dashed line marks the null hypothesis. $A_{\text {diss }}$ denotes amplitude for the spectral distortion template computed for the total distortion signal expected from damping of acoustic modes in $\Lambda \mathrm{CDM}$. $A_{\mathrm{CRR}}$ denotes the amplitude for the spectral distortions induced by recombination of $\mathrm{H}$ and $\mathrm{He}$ (see Section 3.6 for details). For 'Voyage 2050', a scaled version of SuperPIXIE was assumed with $\simeq 5$ times higher sensitivity 
observation even after foreground marginalization (Section 4.2), and thus crosses the critical thresholds for detecting the dissipation $\mu$-distortion and CRR, embracing all expected distortions in the CSM.

\subsection{Foreground challenge for CMB spectral distortion}

Robust detection of spectral distortion signals in the presence of bright astrophysical foregrounds requires observations over multiple decades in frequency, between $\simeq 10 \mathrm{GHz}$ and a few $\times \mathrm{THz}$. Our current understanding of the intensity foregrounds comes primarily from Planck, WMAP and assorted ground-based experiments. At the sensitivities of these observations, the intensity foregrounds could be modeled with sufficient accuracy using a limited set of parameters. We use this foreground parametrization to make spectral distortion forecasts [332]. Figure 9 compares several predicted spectral distortions (e.g., [35], for overview) and the largest astrophysical foregrounds to the sensitivity of possible next-generation spectrometers. At high frequencies, the foregrounds are dominated by dust emission from the Milky Way and the cosmic infrared background, while at low frequencies Galactic synchrotron and free-free emission dominate.

Pioneering steps towards $y \simeq 10^{-7}-10^{-6}$ and technology development may be possible from the ground and balloons, using concepts similar to COSMO, OLIMPO [333, 334], ARCADE [335, 336] and BISOU [337]. However, because the distortions peak at frequencies above $200 \mathrm{GHz}$, broad frequency coverage outside the atmospheric windows ultimately requires a space mission to detect $\mu \simeq 10^{-8}$ or the CRR [275, 276, 313, 332, 338, 339]. To prepare for the analysis of CMB spectral distortions, we will be able to capitalize on existing analysis techniques (e.g., [340]) used in $\mathrm{CMB}$ anisotropy studies, although a new synergistic approach (combining multiple data sets) and observing strategy (e.g., small-patch vs. all-sky) have yet to be fully developed. First steps in this direction were recently taken by [341, 342].

Using the known foreground signals, expected CMB spectral distortions, and realistic frequency coverage and sensitivity estimated from currently existing technologies (see Fig. 9), we produce forecasts for various spectrometer concepts, summarized in Fig. 10. A detailed description of the forecasting method can be found in [332]. The key points are as follows: a pathfinding concept like PRISTINE could detect the relativistic SZ distortion at $\simeq 2 \sigma$, measure the expected $y$-distortion at high significance and deliver an upper limit of $|\mu|<8 \times 10^{-7}$ (95\% c.1.) using readily available technology with only 2 years of integration time. This would already yield important constraints on galactic feedback models (Fig. 8) and also provides us with invaluable information about distortion foregrounds. Should polarization sensitivity be included, these observations could also be used to obtain a cosmic-variancelimited measurement of $\tau$ and further mitigate foregrounds in planned $B$-mode polarization searches [331].

PIXIE's extended low-frequency coverage and enhanced sensitivity produces significant improvements over PRISTINE in detection significances for $k T_{\mathrm{e}}$ and $y$ (see Fig. 10), while improving the upper limit on $\mu$-distortions by an additional order of magnitude to $|\mu|<8 \times 10^{-8}$ (95\% c.l.). The SuperPIXIE concept employs significantly more low-frequency sensitivity and could surpass the threshold for a detection 
of $\mu=2 \times 10^{-8}$ at $3 \sigma$. New information on the complexity of foregrounds could allow for better mission optimization in terms of angular resolution, scan strategy, sensitivity and frequency coverage. With sensitivity improvements over SuperPIXIE by a factor of 5 (ignoring even an optimized frequency sampling from better understanding of the foreground complexity), a Voyage 2050 spectral distortion mission could target a $0.02 \%$ measurement of $y$, a $0.3 \%$ measurement of rSZ, a $10 \%$ or better measurement of $\mu$, and a $10 \%$ measurement of the cosmological recombination lines. This would cross all thresholds for characterization of the distortion expected in the CSM, while covering the full discovery space to new physics (see Fig. 11).

For these estimates, percent-level priors on the low-frequency foregrounds were imposed, anticipating external information from ground-based low-frequency observatories (e.g., C-BASS and S-PASS) to become available. For PRISTINE, this has a significant impact on the forecasted errors, while the other cases are far less affected, suggesting that PRISTINE's science capabilities could be significantly enhanced by adding channels below $v<90 \mathrm{GHz}$. The detection threshold for the CRR is less sensitive to these choices [277].

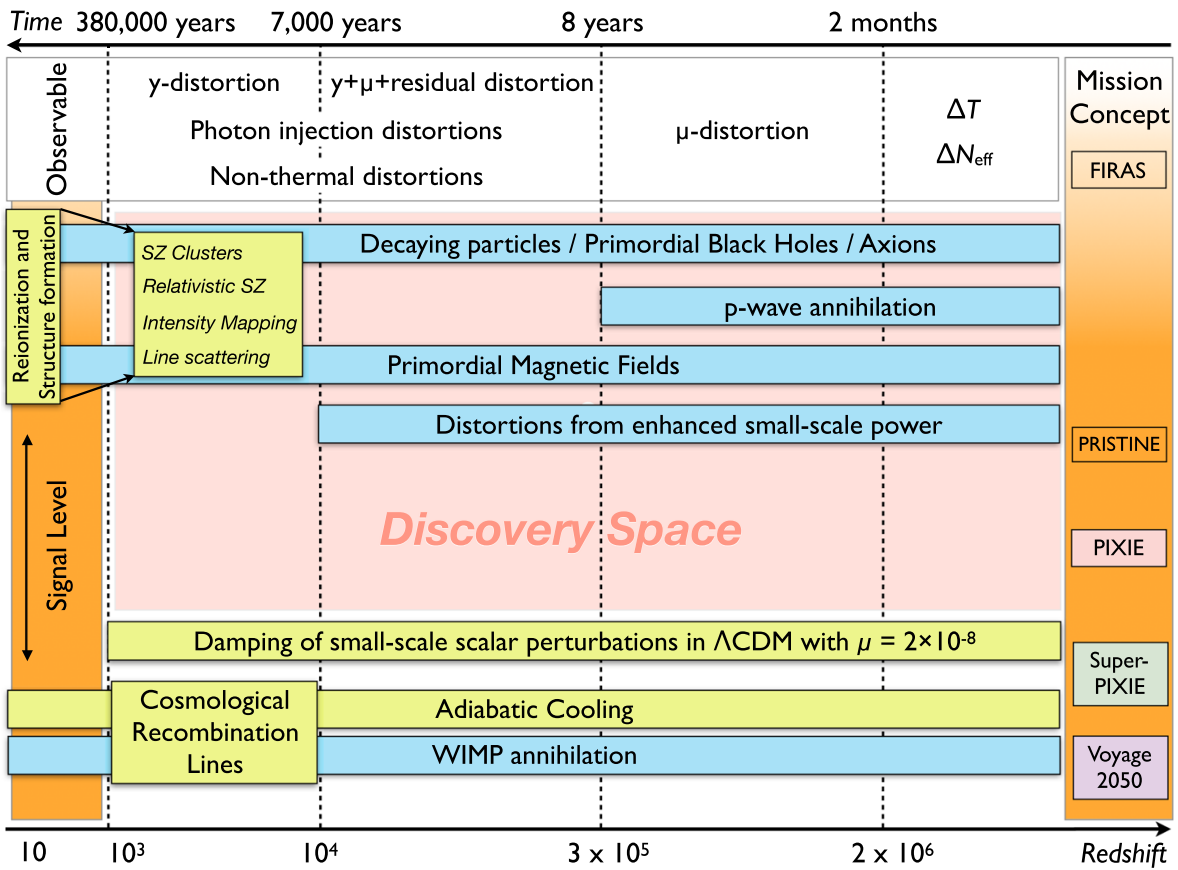

Fig. 11 Science thresholds and mission concepts of increasing sensitivity. Guaranteed sources of distortions and their expected signal levels are shown in yellow), while non-standard processes with possible signal levels are presented in turquoise. Spectral distortions could open a new window to the prerecombination Universe with a vast discovery space to new physics that is accessible on the path towards a detection and characterization of the $\mu$-distortion from the dissipation of small-scale acoustic modes set by inflation and the cosmological recombination radiation 

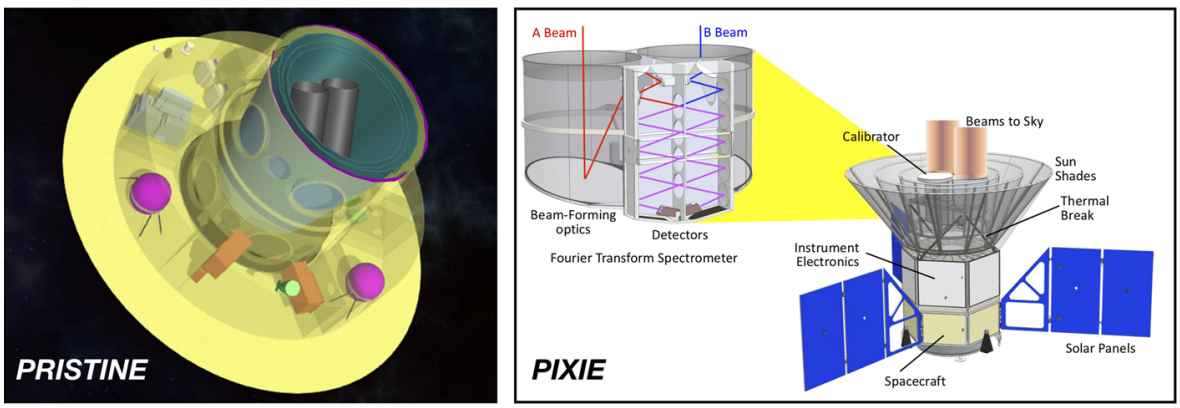

Fig. 12 Illustrations of previous missions. The left panel depicts the observing platform envisioned for PRISTINE, where the two telescopes (each with $\simeq 36 \mathrm{~cm}$ primary mirror) of the FTS are visible in gray [331]. The right panel shows a zoomed in version of the FTS (primary mirrors $\simeq 55 \mathrm{~cm}$ ) alongside the PIXIE spacecraft [41]. For SuperPIXIE, multiple copies of the PIXIE FTS are combined [58]

\subsection{Possible mission concepts and experimental roadmap}

The next frontier in CMB spectroscopy is to detect the tiny departures from a perfect blackbody predicted in the current paradigm (see Fig. 9). This will open a completely new window on cosmology and particle physics, which is within reach of present-day technology, but requires a huge step forward in overall sensitivity from $C O B E$ /FIRAS - ideally a factor of no less than $10^{5}$ (see Fig. 11 for science thresholds). This sensitivity can be achieved using FTS concepts (for illustration see Fig. 12) and we advocate for such a space mission in the ESA science program for 2035-2050.

However, while technically the required improvement of sensitivity seems within reach by 2035 , and possibly even before that, it calls for a dedicated roadmap that builds on the heritage of $C O B E$ /FIRAS but minimizes the risks of too bold a singlestep jump into the unknown. Foregrounds, in particular, are a source of concern. Past experience has shown that each new space mission operating at microwave and submillimeter wavelengths came with surprises concerning galactic and extragalactic foregrounds, resulting in substantial revisions to pre-existing models. So far, all obstacles could be overcome and we are optimistically looking towards future $\mathrm{CMB}$ anisotropy measurements to further our understanding of the Universe. In CMB spectroscopy, it will be crucial to understand how to best deal with those foregrounds, reducing residuals to $0.1 \mathrm{Jy} / \mathrm{sr}$ or better. This will be learnt from improved observations in the next decade. Taking into account programmatic constraints at ESA and potential partners, we advocate for two possible paths forward to achieve the science goals in the ESA Voyage 2050 timeframe.

\subsubsection{L-class mission with pathfinder}

In the first scenario, we envision an ambitious L-class space mission based on a scaled and further optimized version of SuperPIXIE [58] with $\simeq 2-5$ times better sensitivity or more. This could allow a measurement of all the expected primordial distortions, in particular, a $>5 \sigma$ detection of the $\mu$-distortion from dissipation 
and the CRR (see Fig. 10), crossing the threshold to characterizing these signals. In this case, a low-cost pathfinder, similar to PRISTINE [331] and consisting of a single FTS with a sensitivity improved by a factor $\simeq 10^{2}-10^{3}$ over COBE/FIRAS, could be flown in low-Earth orbit around 2025-2030. Even a pathfinder like this would already cross important science thresholds (see Section 4.2, Fig. 10), however, slightly enhanced low-frequency coverage and sensitivity may be desirable (see Section 4.2). Based on the knowledge built in the analysis of the data from the pathfinder, we would further optimize the spectrometer for measuring spectral distortions in the presence of foreground emission. If polarization sensitivity is included, the pathfinder could furthermore complement ongoing searches for primordial $B$ modes, providing extra high frequency coverage and hence significant leverage for foreground removal at large angular scales if flown on until $\simeq 2030$. This information could also be used to explore if polarization sensitivity can help with the cleaning of spectral distortion foregrounds.

In addition to optimizing the L-class spectrometer for foreground removal, one will have to understand how to best make use of data sets that will be available by then. Ground-based observations at low-frequencies $v \lesssim 5 \mathrm{GHz}$, e.g., with CBASS, S-PASS or the SKA, will provide valuable new information to constrain the fluctuating part of the low-frequency foregrounds. The Rubin Observatory (previously known as LSST), Euclid and DESI will have completed their galaxy surveys, allowing to build direct models for extra-galactic foreground signals that can be utilized. SO, CMB-S4 and Litebird are also expected to have completed their observations and in combination with the spectrometer will again allow us to further model and subtract the fluctuating parts of the distortion foregrounds. Combined with the unprecedented control of systematics, frequency coverage and spectral sensitivity of the envisioned spectrometer this will provide us with the necessary tools to tackle the various challenges.

While the required FTS technology has existed for several decades and is well understood, we may need to further explore alternatives to cover the low-frequency end $(v \simeq 5-50 \mathrm{GHz}$ ) of the spectrum. This band yields the largest gains for measuring $\mu$-distortions [332] and spectrometer-on-chip or radiometer designs may perform better. In tandem with the pathfinder, an optimized L-class spectrometer should deliver breakthrough science encompassing all CSM distortions.

\subsubsection{M-class mission within Voyage 2050 program}

The second option would be to fly, preferably by 2030-2035 and thus in collaboration with international partners, an intermediate (e.g., M-class) mission with a spectrometer design closely following SuperPIXIE [58]. Depending on budgetary constraints, the number of FTS replicas can be adjusted, although the main cost driver stems from the cryogenic cooling chain. We expect small changes to the design to be compensated by further optimization of the instrument, so that a mission like this should be able to detect the dissipation $\mu$-distortion at $\simeq 3 \sigma$. Although the $\mu$-detection significance could still suffer from foreground complexities, accurate measurements 
of the low-redshift $y$-distortion and SZ temperature correction are guaranteed. A vast discovery space would furthermore be explored, constraining many new physics examples with significantly improved bounds on $\mu$ (see Fig. 11). In addition, there is a guaranteed scientific harvest from the delivered, absolutely-calibrated all-sky maps at hundreds of frequencies, not only for cosmology but also for many branches of astrophysics (see Section 4.4). Ultimate spectral distortion measurements would then be targeted with an independent future L-class space mission (potentially capitalizing on ideas to go back to the moon [343]) beyond Voyage 2050.

\subsection{Synergies}

A CMB spectrometer will deliver many new constraints for cosmology (e.g., Figs. 10 and 11). Here, we wish to emphasize the synergistic gains from combining it with future $\mathrm{CMB}$ imaging (ground- or space-based), $21 \mathrm{~cm}$ measurements and galaxy surveys. A CMB spectrometer will obtain low-resolution $\left(\delta \theta \simeq 1^{\circ}\right)$, absolutelycalibrated maps of the full sky at hundreds of frequencies. These maps will allow us to calibrate high-resolution CMB imagers to unprecedented levels, opening many possibilities for studying CMB secondaries [59]. They will also help with the cleaning of $\mathrm{CMB}$ foregrounds at the largest angular scales, providing unprecedented control of systematics and allowing us to explore significantly extended foreground parametrizations (e.g., [338]). If polarization sensitivity is included, as in all currently considered spectrometer designs, this would also allow us to obtain a cosmic-variance-limited measurement of $\tau$ and help in further improving constraints on primordial $B$-modes. This, however, requires additional consideration, since it implies reduced spectral distortion sensitivity. ${ }^{4}$

In return, high-resolution CMB imaging with upcoming or planned experiments (e.g., SO, CMB-S4 and Litebird) and low-frequency $(v \lesssim 10 \mathrm{GHz})$ observations by ground-based experiments (e.g., C-BASS, S-PASS and SKA) can provide vital information about the spatially-varying foreground components at small scales. The Rubin Observatory, Euclid and DESI will further help us to improve models of extragalactic foreground signals (e.g., integrated CO emission (e.g., [312, 328, 344])). Combined with the $\mathrm{CMB}$ spectrometer, these will allow us to mitigate many of the foregrounds, enabling us to inch closer to the ultimate goals of detecting and characterizing $\mu$ and the CRR.

A CMB spectrometer will also open the path for many cross-correlation studies with future $21 \mathrm{~cm}$ measurements and galaxy surveys to further improve our understanding of the low-redshift Universe at the largest angular scales [44, 45, 52, 53, 56]. It can also be used to study the origin of large-scale CMB anomalies and isotropy of the Universe [57, 345]. All of the above provide unique scientific opportunities within the ESA Voyage 2050 program that would further our understanding of astrophysics and cosmology.

\footnotetext{
${ }^{4}$ The calibrator is stowed in polarization-mode, when both apertures directly observe the sky.
} 


\section{Conclusions}

CMB spectral distortions probe many processes throughout the history of the Universe. Precision spectroscopy, possible with existing technology, would provide key tests for processes expected within the CSM and open an enormous discovery space to new physics. This offers unique scientific opportunities for furthering our understanding of inflation, recombination, reionization and particle physics (see Section 3 and Fig. 11). Several experimental and theoretical challenges have to be overcome before we can fully exploit this new window to early- and late-Universe physics (see Sections 4.1 and 4.2). However, as highlighted in this contribution, the potential gains are immense and the field is entering a phase of accelerated growth after decades of dormancy. With a coordinated approach, possibly in multiple stages or with one ambitious L-class mission (see Section 4.3 for detailed discussion), we could thus see first precision measurements of some of the fundamental observables of our Universe in the ESA Voyage 2050 program.

We contrasted the scientific outcomes of various mission concepts (see Fig. 10 for detailed comparison of the anticipated distortion constraints). For the most ambitious experimental approach we have shown that beyond a significant detection of the inflationary $\mu$ distortion signal one can begin to characterize the CRR. This would allow to directly probe the cosmological recombination process occurring some 400,000 years after the Big Bang and deliver the most pristine way of measuring the primordial helium abundance and baryonic content of the Universe. World-wide this would be a unique initiative for advancing the long-standing legacy of $C O B E / F I R A S$ all the way to one of the smallest expected distortion signals produced in the early Universe.

Acknowledgements We cordially thank the contributors to the Decadal White Paper [82], which provided the starting point for this substantially extended response to the ESA Voyage 2050 call. This work has received funding from the European Research Council under the European Union's Horizon 2020 research and innovation program (grant agreement No 725456, CMBSPEC) as well as the Royal Society (grants UF130435 and RG140523). JARM acknowledges financial support from the Spanish Ministry of Science and Innovation (MICINN) under the project AYA2017-84185-P, and from the European Union's Horizon 2020 research and innovation programme under Grant Agreement 687312 (RADIOFOREGROUNDS). C.H.-M. acknowledges the support of the Spanish Ministry of Science through project PGC2018-097585B-C21. Financial support from the ASI/Physics Department of the University of Roma-Tor Vergata agreement n. 2016-24-H.0 for study activities of the Italian cosmology community is acknowledged.

Open Access This article is licensed under a Creative Commons Attribution 4.0 International License, which permits use, sharing, adaptation, distribution and reproduction in any medium or format, as long as you give appropriate credit to the original author(s) and the source, provide a link to the Creative Commons licence, and indicate if changes were made. The images or other third party material in this article are included in the article's Creative Commons licence, unless indicated otherwise in a credit line to the material. If material is not included in the article's Creative Commons licence and your intended use is not permitted by statutory regulation or exceeds the permitted use, you will need to obtain permission directly from the copyright holder. To view a copy of this licence, visit http://creativecommons.org/licenses/by/4.0/.

\section{References}

1. Mather, J.C. et al.: ApJ 420, 439 (1994). https://doi.org/10.1086/173574

2. Fixsen, D.J. et al.: ApJ 473, 576 (1996). https://doi.org/10.1086/178173 
3. Smoot, G.F. et al.: ApJL 396, L1 (1992). https://doi.org/10.1086/186504

4. Bennett, C.L. et al.: ApJS 148, 1 (2003)

5. Planck Collaboration: A\&A 571, A16 (2014). https://doi.org/10.1051/0004-6361/201321591

6. Fixsen, D.J.: ApJ 707, 916 (2009). https://doi.org/10.1088/0004-637X/707/2/916

7. Silk, J.: J. Chluba, Science 344, 586 (2014). https://doi.org/10.1126/science.1252724

8. Zeldovich, Y.B., Sunyaev, R.A.: ApSS 4, 301 (1969). https://doi.org/10.1007/BF00661821

9. Sunyaev, R.A., Zeldovich, Y.B.: ApSS 7, 20 (1970). https://doi.org/10.1007/BF00653472

10. Illarionov, A.F., Sunyaev, R.A.: Sov. Astron. 18, 691 (1975)

11. Burigana, C., Danese, L., de Zotti, G.: A\&A 246, 49 (1991)

12. Hu, W., Silk, J.: Phys. Rev. D 48, 485 (1993). https://doi.org/10.1103/PhysRevD.48.485

13. Chluba, J., Sunyaev, R.A.: MNRAS 419, 1294 (2012). https://doi.org/10.1111/j.1365-2966.2011. 19786.x

14. Khatri, R., Sunyaev, R.A.: JCAP 9, 016 (2012). https://doi.org/10.1088/1475-7516/2012/09/016

15. Chluba, J.: MNRAS 434, 352 (2013). https://doi.org/10.1093/mnras/stt1025

16. Chluba, J.: MNRAS 436, 2232 (2013). https://doi.org/10.1093/mnras/stt1733

17. Chluba, J., Jeong, D.: MNRAS 438, 2065 (2014). https://doi.org/10.1093/mnras/stt2327

18. Chluba, J.: MNRAS 454, 4182 (2015). https://doi.org/10.1093/mnras/stv2243

19. Dubrovich, V.K.: Sov. Astron. Lett. 1, 196 (1975)

20. Sunyaev, R.A.: J. Chluba, AN 330, 657 (2009). https://doi.org/10.1002/asna.200911237

21. Chluba, J., Ali-Haïmoud, Y.: MNRAS 456, 3494 (2016). https://doi.org/10.1093/mnras/stv2691

22. Lyubarsky, Y.E., Sunyaev, R.A.: A\&A 123, 171 (1983)

23. Chluba, J., Sunyaev, R.A.: A\&A 501, 29 (2009). https://doi.org/10.1051/0004-6361/200809840

24. Chluba, J.: MNRAS 402, 1195 (2010). https://doi.org/10.1111/j.1365-2966.2009.15957.x

25. Enßlin, T.A., Kaiser, C.R.: A\&A 360, 417 (2000)

26. Slatyer, T.R.: vol. D93. https://doi.org/10.1103/PhysRevD.93.023521 (2016)

27. Acharya, S.K., Khatri, R. Phys. Rev. D 99(4), 043520 (2019). https://doi.org/10.1103/PhysRevD. 99.043520

28. Sunyaev, R.A., Zeldovich, Y.B.: Comments Astrophys. Space Phys. 2, 66 (1970)

29. Sunyaev, R.A., Zeldovich, I.B.: ARA\&A 18, 537 (1980). https://doi.org/10.1146/annurev.aa.18. 090180.002541

30. Sunyaev, R.A., Khatri, R.: IJMPD 1330014, 22 (2013). https://doi.org/10.1142/S0218271813300140

31. André, P. et al.: JCAP 2, 006 (2014). https://doi.org/10.1088/1475-7516/2014/02/006

32. Chluba, J.: ArXiv e-prints (2014)

33. Tashiro, H.: Prog. of Theo. Exp. Phys. 2014(6), 06B107. https://doi.org/10.1093/ptep/ptu066 (2014)

34. De Zotti, G., Negrello, M., Castex, G., Lapi, A., Bonato, M.: JCAP 3, 047 (2016). https://doi.org/ 10.1088/1475-7516/2016/03/047

35. Chluba, J.: MNRAS 460, 227 (2016). https://doi.org/10.1093/mnras/stw945

36. Chluba, J.: arXiv:1806.02915 (2018)

37. Kogut, A. et al.: ApJ 734, 4 (2011). https://doi.org/10.1088/0004-637X/734/1/4

38. Mukherjee, S., Silk, J., Wandelt, B.D.: MNRAS 477(4), 4473 (2018). https://doi.org/10.1093/mnras/ sty 888

39. Mukherjee, S., Silk, J., Wandelt, B.D.: Phys. Rev. D 100(10), 103508 (2019). https://doi.org/10.1103/ PhysRevD.100.103508

40. Kogut, A. et al.: JCAP 7, 25 (2011). https://doi.org/10.1088/1475-7516/2011/07/025

41. Kogut, A., et al.: In: SPIE Conference Series. Proc. SPIE, vol. 9904 (2016), Proc. SPIE, vol. 9904, 99040W. https://doi.org/10.1117/12.2231090

42. Sunyaev, R.A., Zeldovich, Y.B.: Comments Astrophys. Space Phys. 4, 173 (1972)

43. Carlstrom, J.E., Holder, G.P., Reese, E.D.: ARA\&A 40, 643 (2002). https://doi.org/10.1146/annurev. astro.40.060401.093803

44. Pajer, E., Zaldarriaga, M.: Phys. Rev. Lett 109(2), 021302 (2012). https://doi.org/10.1103/ PhysRevLett.109.021302

45. Ganc, J., Komatsu, E.: Phys. Rev. D 86(2), 023518 (2012). https://doi.org/10.1103/PhysRevD.86. 023518

46. Emami, R. et al.: Phys. Rev. D91(12) 123531. https://doi.org/10.1103/PhysRevD.91.123531 (2015)

47. Ota, A.: Phys. Rev. D94, 103520 (2016). https://doi.org/10.1103/PhysRevD.94.103520

48. Danese, L., de Zotti, G.: A\&A 94(2), L33 (1981)

49. Balashev, S.A. et al.: ApJ 810(2), 131 (2015). https://doi.org/10.1088/0004-637X/810/2/131

50. Burigana, C. et al.: JCAP 2018(4), 021 (2018). https://doi.org/10.1088/1475-7516/2018/04/021 
51. Trombetti, T., Burigana, C., Chierici, F.: arXiv:2007.02292 (2020)

52. Refregier, A., Komatsu, E., Spergel, D.N., Pen, U.L.: Phys. Rev. D 61(12), 123001 (2000). https://doi.org/10.1103/PhysRevD.61.123001

53. Zhang, P., Pen, U.L., Trac, H.: MNRAS 355, 451 (2004). https://doi.org/10.1111/j.1365-2966.2004. 08328.x

54. Pitrou, C., Bernardeau, F., Uzan, J.P.: JCAP 7, 19 (2010). https://doi.org/10.1088/1475-7516/ 2010/07/019

55. Hill, J.C., Pajer, E.: Phys. Rev. D88(6) 063526. https://doi.org/10.1103/PhysRevD.88.063526 (2013)

56. Alvarez, M.A.: ApJ 824(2), 118 (2016). https://doi.org/10.3847/0004-637X/824/2/118

57. Dai, L., Jeong, D., Kamionkowski, M., Chluba, J.: Phys. Rev. D 87(12), 123005 (2013). https:// doi.org/10.1103/PhysRevD.87.123005

58. Kogut, A., Abitbol, M.H., Chluba, J., Delabrouille, J., Fixsen, D., Hill, J.C., Patil, S.P., Rotti, A.: In: BAAS, vol. 51, pp. 113. (2019)

59. Basu, K., Remazeilles, M., Melin, J.B., Alonso, D., Bartlett, J.G., Battaglia, N., Chluba, J., Churazov, E., Delabrouille, J., Erler, J., Ferraro, S., Hernández-Monteagudo, C., Hill, J.C., Hotinli, S.C., Khabibullin, I., Madhavacheril, M., Mroczkowski, T., Nagai, D., Raghunathan, S., Rubino Martin, J.A., Sayers, J., Scott, D., Sugiyama, N., Sunyaev, R., Zubeldia, Í.: arXiv:1909.01592 (2019)

60. Calabrese, E., Alonso, D., Dunkley, J.: Phys. Rev. D 95(6), 063504 (2017). https://doi.org/10.1103/ PhysRevD.95.063504

61. Planck Collaboration, Aghanim, N., Akrami, Y., Ashdown, M., Aumont, J., Baccigalupi, C., Ballardini, M., Banday, A.J., Barreiro, R.B., Bartolo, N., Basak, S., Battye, R., Benabed, K., Bernard, J.P., Bersanelli, M., Bielewicz, P., Bock, J.J., Bond, J.R., Borrill, J., Bouchet, F.R., Boulanger, F., Bucher, M., Burigana, C., Butler, R.C., Calabrese, E., Cardoso, J.F., Carron, J., Challinor, A., Chiang, H.C., Chluba, J., Colombo, L.P.L., Combet, C., Contreras, D., Crill, B.P., Cuttaia, F., de Bernardis, P., G. de Zotti, P., Delabrouille, J., Delouis, J.M., Di Valentino, E., Diego, J.M., Doré, O., Douspis, M., Ducout, A., Dupac, X., Dusini, S., Efstathiou, G., Elsner, F., Enßlin, T.A., Eriksen, H.K., Fantaye, Y., Farhang, M., Fergusson, J., Fernandez-Cobos, R., Finelli, F., Forastieri, F., Frailis, M., Fraisse, A.A., Franceschi, E., Frolov, A., Galeotta, S., Galli, S., Ganga, K., Génova-Santos, R.T., Gerbino, M., Ghosh, T., González-Nuevo, J., Górski, K.M., Gratton, S., Gruppuso, A., Gudmundsson, J.E., Hamann, J., Handley, W., Hansen, F.K., Herranz, D., Hildebrandt, S.R., Hivon, E., Huang, Z., Jaffe, A.H., Jones, W.C., Karakci, A., Keihänen, E., Keskitalo, R., Kiiveri, K., Kim, J., Kisner, T.S., Knox, L., Krachmalnicoff, N., Kunz, M., Kurki-Suonio, H., Lagache, G., Lamarre, J.M., Lasenby, A., Lattanzi, M., Lawrence, C.R., Le Jeune, M., Lemos, P., Lesgourgues, J., Levrier, F., Lewis, A., Liguori, M., Lilje, P.B., Lilley, M., Lindholm, V., López-Caniego, M., Lubin, P.M., Ma, Y.Z., Macías-Pérez, J.F., Maggio, G., Maino, D., Mandolesi, N., Mangilli, A., Marcos-Caballero, A., Maris, M., Martin, P.G., Martinelli, M., Martínez-González, E., Matarrese, S., Mauri, N., McEwen, J.D., Meinhold, P.R., Melchiorri, A., Mennella, A., Migliaccio, M., Millea, M., Mitra, S., Miville-Deschênes, M.A., Molinari, D., Montier, L., Morgante, G., Moss, A., Natoli, P., Nørgaard-Nielsen, H.U., Pagano, L., Paoletti, D., Partridge, B., Patanchon, G., Peiris, H.V., Perrotta, F., Pettorino, V., Piacentini, F., Polastri, L., Polenta, G., Puget, J.L., Rachen, J.P., Reinecke, M., Remazeilles, M., Renzi, A., Rocha, G., Rosset, C., Roudier, G., Rubiño-Martín, J.A., Ruiz-Granados, B., Salvati, L., Sandri, M., Savelainen, M., Scott, D., Shellard, E.P.S., Sirignano, C., Sirri, G., Spencer, L.D., Sunyaev, R., Suur-Uski, A.S., Tauber, J.A., Tavagnacco, D., Tenti, M., Toffolatti, L., Tomasi, M., Trombetti, T., Valenziano, L., Valiviita, J., Van Tent, B., Vibert, L., Vielva, P., Villa, F., Vittorio, N., Wand elt, B.D., Wehus, I.K., White, M., White, S.D.M., Zacchei, A., Zonca, A.: arXiv:1807.06209 (2018)

62. Starobinsky, A.A.: Phys. Lett. B91, 99 (1980). https://doi.org/10.1016/0370-2693(80)90670-X

63. Guth, A.H.: Phys. Rev. D23, 347 (1981). https://doi.org/10.1103/PhysRevD.23.347

64. Linde, A.D.: Phys. Lett. 108B, 389 (1982). https://doi.org/10.1016/0370-2693(82)91219-9

65. Albrecht, A., Steinhardt, P.J.: Phys. Rev. Lett. 48, 1220 (1982). https://doi.org/10.1103/PhysRevLett. 48.1220

66. Mukhanov, V.F., Chibisov, G.V.: SJETP Lett. 33, 532 (1981)

67. Hawking, S.W.: Phys. Lett. 115B, 295 (1982). https://doi.org/10.1016/0370-2693(82)90373-2

68. Starobinsky, A.A.: JETP Lett. 30, 682 (1979)

69. Guth, A.H., Pi, S.Y.: Phys. Rev. Lett. 49, 1110 (1982). https://doi.org/10.1103/PhysRevLett.49.1110

70. Gasperini, M., Veneziano, G.: Astropart. Phys. 1, 317 (1993). https://doi.org/10.1016/0927-6505(93) 90017-8

71. Wands, D.: Phys. Rev. 023507, D60 (1999). https://doi.org/10.1103/PhysRevD.60.023507 
72. Khoury, J., Ovrut, B.A., Seiberg, N., Steinhardt, P.J., Turok, N.: Phys. Rev. 086007, D65 (2002). https://doi.org/10.1103/PhysRevD.65.086007

73. Hollands, S., Wald, R.M.: Gen. Rel. Grav. 34, 2043 (2002). https://doi.org/10.1023/A:1021175 216055

74. Brandenberger, R.H., Nayeri, A., Patil, S.P., Vafa, C.: Int. J. Mod. Phys. A22, 3621 (2007). https:// doi.org/10.1142/S0217751X07037159

75. Craps, B., Hertog, T., Turok, N.: Phys. Rev. 043513, D86 (2012). https://doi.org/10.1103/PhysRevD. 86.043513

76. Creminelli, P., Nicolis, A.: E. Trincherini, JCAP 1011, 021 (2010). https://doi.org/10.1088/1475$7516 / 2010 / 11 / 021$

77. Ijjas, A., Steinhardt, P.J.: Phys. Rev. Lett. 117(12), 121304 (2016). https://doi.org/10.1103/ PhysRevLett.117.121304

78. Dobre, D.A. et al.: JCAP 2018(3), 020 (2018). https://doi.org/10.1088/1475-7516/2018/03/020

79. Ade, P.A.R. et al.: Submitted to Phys. Rev. Lett. (2018)

80. Bezrukov, F., Shaposhnikov, M.: Phys. Lett. B659, 703 (2008). https://doi.org/10.1016/j.physletb. 2007.11.072

81. Byrnes, C.T., Cole, P.S., Patil, S.P. (2018)

82. Chluba, J. et al.: BAAS 51(3), 184 (2019)

83. Sunyaev, R.A., Zeldovich, Y.B.: ApSS 9, 368 (1970). https://doi.org/10.1007/BF00649577

84. Daly, R.A.: ApJ 371, 14 (1991). https://doi.org/10.1086/169866

85. Hu, W., Silk, J., Scott, D.: ApJL 430, L5 (1994). https://doi.org/10.1086/187424

86. Chluba, J., Khatri, R., Sunyaev, R.A.: MNRAS 425, 1129 (2012). https://doi.org/10.1111/j.13652966.2012.21474.x

87. Khatri, R., Sunyaev, R.A.: J. Chluba, A\&A 543, A136 (2012). https://doi.org/10.1051/0004-6361/ 201219590

88. Chluba, J., Erickcek, A.L., Ben-Dayan, I.: ApJ 758, 76 (2012). https://doi.org/10.1088/0004-637X/ $758 / 2 / 76$

89. Chluba, J., Hamann, J., Patil, S.P.: IJMP D 1530023, 24 (2015). https://doi.org/10.1142/S02182718 15300232

90. Cabass, G., Melchiorri, A., Pajer, E.: Phys. Rev. D93(8) 083515. https://doi.org/10.1103/PhysRevD. 93.083515 (2016)

91. Pajer, E., Zaldarriaga, M.: JCAP 2, 036 (2013). https://doi.org/10.1088/1475-7516/2013/02/036

92. Khatri, R., Sunyaev, R.A.: JCAP 6, 026 (2013). https://doi.org/10.1088/1475-7516/2013/06/026

93. Powell, B.A.: ArXiv e-prints (2012)

94. Clesse, S., Garbrecht, B., Zhu, Y.: JCAP 2014, 046 (2014). https://doi.org/10.1088/1475-7516/2014/ $10 / 046$

95. Cabass, G., Di Valentino, E., Melchiorri, A., Pajer, E., Silk, J.: Phys. Rev. D94(2) 023523. https://doi.org/10.1103/PhysRevD.94.023523 (2016)

96. Inomata, K., Nakama, T.: Phys. Rev. D 99(4), 043511 (2019). https://doi.org/10.1103/PhysRevD. 99.043511

97. Starobinsky, A.A.: JETP Lett. 55, 489 (1992). [Pisma Zh. Eksp. Teor. Fiz. 55, 477(1992)]

98. Adams, J.A., Cresswell, B., Easther, R.: Phys. Rev. 123514, D64 (2001). https://doi.org/10.1103/ PhysRevD.64.123514

99. Hazra, D.K., Shafieloo, A., Smoot, G.F., Starobinsky, A.A.: Phys. Rev. Lett. 113(7), 071301 (2014). https://doi.org/10.1103/PhysRevLett.113.071301

100. Polnarev, A.G., Musco, I.: Class. Quant. Grav. 24, 1405 (2007). https://doi.org/10.1088/0264-9381/ $24 / 6 / 003$

101. Kohri, K., Lyth, D.H., Melchiorri, A.: JCAP 2008(4), 038 (2008). https://doi.org/10.1088/14757516/2008/04/038

102. Ben-Dayan, I., Brustein, R.: JCAP 2010, 007 (2010). https://doi.org/10.1088/1475-7516/2010/09/007

103. Choudhury, S., Mazumdar, A.: Phys. Lett. B733, 270 (2014). https://doi.org/10.1016/j.physletb. 2014.04.050

104. Clesse, S., García-Bellido, J.: Phys. Rev. D92(2) 023524. https://doi.org/10.1103/PhysRevD. $92.023524(2015)$

105. Germani, C., Prokopec, T.: Phys. Dark Univ. 18, 6 (2017). https://doi.org/10.1016/j.dark.2017. 09.001

106. Barnaby, N., Huang, Z.: Phys. Rev. 126018, D80 (2009). https://doi.org/10.1103/PhysRevD. 80.126018 
107. Cook, J.L., Sorbo, L.: Phys. Rev. D85, 023534 (2012). https://doi.org/10.1103/PhysRevD. 86.069901, https://doi.org/10.1103/PhysRevD.85.023534

108. Battefeld, D., Battefeld, T., Fiene, D.: Phys. Rev. D89(12) 123523. https://doi.org/10.1103/ PhysRevD.89.123523 (2014)

109. Dimastrogiovanni, E., Fasiello, M., Fujita, T.: JCAP 2017, 019 (2017). https://doi.org/10.1088/14757516/2017/01/019

110. Domcke, V., Mukaida, K.: JCAP 1811(11), 020 (2018). https://doi.org/10.1088/1475-7516/ 2018/11/020

111. Linde, A.D.: Phys. Rev. D49, 748 (1994). https://doi.org/10.1103/PhysRevD.49.748

112. Lyth, D.H., Stewart, E.D.: Phys. Rev. D54, 7186 (1996). https://doi.org/10.1103/PhysRevD.54.7186

113. García-Bellido, J.: A. Linde, D. Wands, Phys. Rev. D54, 6040 (1996). https://doi.org/10.1103/ PhysRevD.54.6040

114. Abolhasani, A.A., Firouzjahi, H., Namjoo, M.H.: Class. Quant. Grav. 28, 075009 (2011). https:// doi.org/10.1088/0264-9381/28/7/075009

115. Clesse, S., Garbrecht, B., Zhu, Y.: JCAP 1410(10), 046 (2014). https://doi.org/10.1088/1475-7516/ 2014/10/046

116. Barnaby, N., Peloso, M.: Phys. Rev. Lett. 181301, 106 (2011). https://doi.org/10.1103/PhysRevLett. 106.181301

117. Barnaby, N., Pajer, E., Peloso, M.: Phys. Rev. 023525, D85 (2012). https://doi.org/10.1103/ PhysRevD.85.023525

118. Meerburg, P.D.: E. Pajer, JCAP 1302, 017 (2013). https://doi.org/10.1088/1475-7516/2013/02/017

119. Nakama, T., Chluba, J., Kamionkowski, M.: Phys. Rev. D95(12) 121302. https://doi.org/10.1103/ PhysRevD.95.121302 (2017)

120. Diacoumis, J.A.D., Wong, Y.Y.Y.: JCAP 9, 011 (2017). https://doi.org/10.1088/1475-7516/2017/ 09/011

121. Cho, K., Hong, S.E., Stewart, E.D., Zoe, H.: JCAP 2017, 002 (2017). https://doi.org/10.1088/14757516/2017/08/002

122. Ota, A., Takahashi, T., Tashiro, H., Yamaguchi, M.: JCAP 10, 029 (2014). https://doi.org/10.1088/ 1475-7516/2014/10/029

123. Chluba, J., Dai, L., Grin, D., Amin, M.A., Kamionkowski, M.: MNRAS 446, 2871 (2015). https:// doi.org/10.1093/mnras/stu2277

124. Hu, W.: N. Sugiyama, ApJ 436, 456 (1994). https://doi.org/10.1086/174921

125. Dent, J.B., Easson, D.A., Tashiro, H.: Phys. Rev. D86(2) 023514. https://doi.org/10.1103/PhysRevD. $86.023514(2012)$

126. Chluba, J., Grin, D.: MNRAS 434, 1619 (2013). https://doi.org/10.1093/mnras/stt1129

127. Haga, T., Inomata, K., Ota, A., Ravenni, A.: JCAP 2018, 036 (2018). https://doi.org/10.1088/1475$7516 / 2018 / 08 / 036$

128. Fu, H., Lucca, M., Galli, S., Battistelli, E.S., Hooper, D.C., Lesgourgues, J., Schöneberg, N. (2020)

129. Ade, P. et al.: JCAP 2019(2), 056 (2019). https://doi.org/10.1088/1475-7516/2019/02/056

130. Suzuki, A. et al.: J. Low Temp. Phys. https://doi.org/10.1007/s10909-018-1947-7 (2018)

131. Biagetti, M., Perrier, H., Riotto, A., Desjacques, V.: Phys. Rev. D87(6) 063521. https://doi.org/ 10.1103/PhysRevD.87.063521 (2013)

132. Ota, A., Sekiguchi, T., Tada, Y., Yokoyama, S.: JCAP 2015, 013 (2015). https://doi.org/10.1088/ 1475-7516/2015/03/013

133. Khatri, R., Sunyaev, R.: JCAP 9, 026 (2015). https://doi.org/10.1088/1475-7516/2015/09/026

134. Chluba, J., Dimastrogiovanni, E., Amin, M.A., Kamionkowski, M.: MNRAS 466, 2390 (2017). https://doi.org/10.1093/mnras/stw3230

135. Ota, A.: Phys. Rev. D94(10) 103520. https://doi.org/10.1103/PhysRevD.94.103520 (2016)

136. Ravenni, A., Liguori, M., Bartolo, N., Shiraishi, M.: JCAP 9, 042 (2017). https://doi.org/10.1088/ 1475-7516/2017/09/042

137. Cabass, G., Pajer, E., van der Woude, D.: JCAP 1808(08), 050 (2018). https://doi.org/10.1088/14757516/2018/08/050

138. Dimastrogiovanni, E., Emami, R.: JCAP 12, 015 (2016). https://doi.org/10.1088/1475-7516/2016/ $12 / 015$

139. Akrami, Y.: et al. (2019)

140. Bartolo, N., Liguori, M., Shiraishi, M.: JCAP 1603(03), 029 (2016). https://doi.org/10.1088/1475$7516 / 2016 / 03 / 029$ 
141. Becker, A., Huterer, D.: Phys. Rev. Lett. 121302, 109 (2012). https://doi.org/10.1103/PhysRevLett. 109.121302

142. Oppizzi, F., Liguori, M., Renzi, A., Arroja, F., Bartolo, N.: JCAP 1805(05), 045 (2018). https:// doi.org/10.1088/1475-7516/2018/05/045

143. Remazeilles, M., Chluba, J.: MNRAS 478(1), 807 (2018). https://doi.org/10.1093/mnras/sty1034

144. Hanany, S. et al.: arXiv:1902.10541 (2019)

145. Remazeilles, M., Delabrouille, J., Cardoso, J.F.: MNRAS 410(4), 2481 (2011). https://doi.org/ 10.1111/j.1365-2966.2010.17624.x

146. Camera, S., Santos, M.G., Maartens, R.: MNRAS 448(2), 1035 (2015). https://doi.org/10.1093/ mnras/stv040

147. Doré, O. et al.: arXiv:1412.4872 (2014)

148. Dalal, N., Dore, O., Huterer, D., Shirokov, A.: Phys. Rev. 123514, D77 (2008). https://doi.org/ 10.1103/PhysRevD.77.123514

149. Matarrese, S., Verde, L.: Astrophys. J. 677, L77 (2008). https://doi.org/10.1086/587840

150. Maldacena, J.M.: JHEP 05, 013 (2003). https://doi.org/10.1088/1126-6708/2003/05/013

151. Kawasaki, M., Kohri, K., Moroi, T.: Phys. Rev. D71(8) 083502. https://doi.org/10.1103/PhysRevD. $71.083502(2005)$

152. Kawasaki, M., Kohri, K., Moroi, T., Takaesu, Y.: Phys. Rev. D97(2) 023502. https://doi.org/10.1103/ PhysRevD.97.023502 (2018)

153. Ahmed, Z. et al.: Science 327, 1619 (2010). https://doi.org/10.1126/science. 1186112

154. Aprile, E. et al.: Phys. Rev. Lett. 181301, 109 (2012). https://doi.org/10.1103/PhysRevLett.109. 181301

155. Angloher, G. et al.: Eur. Phys. J. C76(1), 25 (2016). https://doi.org/10.1140/epjc/s10052-016-3877-3

156. Agnese, R., et al.: Phys. Rev. Lett. 116(7), 071301 (2016). https://doi.org/10.1103/PhysRevLett. 116.071301

157. Tan, A., et al.: Phys. Rev. Lett. 117(12), 121303 (2016). https://doi.org/10.1103/PhysRevLett.117. 121303

158. Akerib, D.S., et al.: Phys. Rev. Lett 118(2), 021303 (2017). https://doi.org/10.1103/PhysRevLett. 118.021303

159. Jungman, G., Kamionkowski, M., Griest, K.: Phys. Rep. 267, 195 (1996). https://doi.org/10.1016/ 0370-1573(95)00058-5

160. Feng, J.L., Rajaraman, A., Takayama, F.: Phys. Rev. Lett. 91(1), 011302 (2003). https://doi.org/ 10.1103/PhysRevLett.91.011302

161. Feng, J.L., Rajaraman, A., Takayama, F.: Phys. Rev. D68(6) 063504. https://doi.org/10.1103/ PhysRevD.68.063504 (2003)

162. Kusenko, A.: Phys. Rep. 481, 1 (2009). https://doi.org/10.1016/j.physrep.2009.07.004

163. Feng, J.L.: ARA\&A 48, 495 (2010). https://doi.org/10.1146/annurev-astro-082708-101659

164. Carr, B.J., Kohri, K., Sendouda, Y., Yokoyama, J.: Phys. Rev. D81(10) 104019. https://doi.org/ 10.1103/PhysRevD.81.104019 (2010)

165. Marsh, D.J.E.: Phys. Rep. 643, 1 (2016). https://doi.org/10.1016/j.physrep.2016.06.005

166. Ellis, J., Gelmini, G.B., Lopez, J.L., Nanopoulos, D.V., Sarkar, S.: Nu. Phys. B 373, 399 (1992). https://doi.org/10.1016/0550-3213(92)90438-H

167. Adams, J.A., Sarkar, S., Sciama, D.W.: MNRAS 301, 210 (1998). https://doi.org/10.1046/j.13658711.1998.02017.x

168. Chen, X., Kamionkowski, M.: Phys. Rev. D70(4) 043502. https://doi.org/10.1103/PhysRevD.70. 043502 (2004)

169. Padmanabhan, N., Finkbeiner, D.P.: Phys. Rev. D72(2) 023508. https://doi.org/10.1103/PhysRevD. $72.023508(2005)$

170. Galli, S., Iocco, F., Bertone, G., Melchiorri, A.: Phys. Rev. D 80(2), 023505 (2009). https://doi.org/ 10.1103/PhysRevD.80.023505

171. Slatyer, T.R., Padmanabhan, N., Finkbeiner, D.P.: Phys. Rev. D80(4) 043526. https://doi.org/ 10.1103/PhysRevD.80.043526 (2009)

172. Slatyer, T.R., Wu, C.L.: Phys. Rev. D95(2) 023010. https://doi.org/10.1103/PhysRevD.95.023010 (2017)

173. Poulin, V., Lesgourgues, J., Serpico, P.D.: JCAP 3, 043 (2017). https://doi.org/10.1088/1475-7516/ 2017/03/043

174. Wilkinson, R.J., Bœhm, C.: J. Lesgourg. JCAP 2014, 011 (2014). https://doi.org/10.1088/1475$7516 / 2014 / 05 / 011$ 
175. Dvorkin, C., Blum, K., Kamionkowski, M.: Phys. Rev. D89(2) 023519. https://doi.org/10.1103/ PhysRevD.89.023519 (2014)

176. Wilkinson, R.J., Lesgourgues, J.: C. Bœ,hm, JCAP 2014, 026 (2014). https://doi.org/10.1088/14757516/2014/04/026

177. Gluscevic, V., Boddy, K.K.: Phys. Rev. Lett. 121, 081301 (2018). https://doi.org/10.1103/ PhysRevLett.121.081301

178. Boddy, K.K., Gluscevic, V.: Phys. Rev. D98(8) 083510. https://doi.org/10.1103/PhysRevD.98. $083510(2018)$

179. Jedamzik, K.: JCAP 3, 008 (2008). https://doi.org/10.1088/1475-7516/2008/03/008

180. Poulin, V., Serpico, P.D.: Phys. Rev. Lett. 091101, 114 (2015). https://doi.org/10.1103/PhysRevLett. 114.091101

181. Sarkar, S., Cooper, A.M.: Phys. Lett. B 148, 347 (1984). https://doi.org/10.1016/0370-2693(84) 90101-1

182. Ellis, J., Nanopoulos, D.V., Sarkar, S.: Nuclear Phys. B 259, 175 (1985). https://doi.org/10.1016/ 0550-3213(85)90306-2

183. Kawasaki, M., Sato, K.: Phys. Lett. B 169, 280 (1986). https://doi.org/10.1016/0370-2693(86) 90666-0

184. Hu, W., Silk, J.: Phys. Rev. Lett. 70, 2661 (1993). https://doi.org/10.1103/PhysRevLett.70.2661

185. Aalberts, J.L. et al.: Phys. Rev. D98(2) 023001. https://doi.org/10.1103/PhysRevD.98.023001 (2018)

186. Lucca, M., Schöneberg, N., Hooper, D.C., Lesgourgues, J.: J. Chluba JCAP 02, 026 (2020). https://doi.org/10.1088/1475-7516/2020/02/026

187. Chluba, J., Ravenni, A., Acharya, S.K.: arXiv:2005.11325 (2020)

188. McDonald, P., Scherrer, R.J., Walker, T.P.: Phys. Rev. D63(2) 023001. https://doi.org/10.1103/ PhysRevD.63.023001 (2001)

189. Tashiro, H., Silk, J., Marsh, D.J.E.: Phys. Rev. D88(12) 125024. https://doi.org/10.1103/PhysRevD. 88.125024 (2013)

190. Ejlli, D., Dolgov, A.D.: Phys. Rev. 063514, D90 (2014). https://doi.org/10.1103/PhysRevD. 90.063514

191. Mukherjee, S., Khatri, R., Wandelt, B.D.: JCAP 4, 045 (2018). https://doi.org/10.1088/1475-7516/ 2018/04/045

192. Dimastrogiovanni, E., Krauss, L.M., Chluba, J.: Phys. Rev. D94(2) 023518. https://doi.org/10.1103/ PhysRevD.94.023518 (2016)

193. Ostriker, J.P., Thompson, C.: ApJL 323, L97 (1987). https://doi.org/10.1086/185065

194. Tashiro, H., Sabancilar, E., Vachaspati, T.: JCAP 8, 035 (2013). https://doi.org/10.1088/1475-7516/ 2013/08/035

195. Ali-Haïmoud, Y., Chluba, J., Kamionkowski, M.: Phys. Rev. D115, 071304 (2015). https://doi.org/ 10.1103/PhysRevLett.115.071304

196. Kumar, S., et al. Phys. Rev. D 99(2), 023521 (2019). https://doi.org/10.1103/PhysRevD.99.023521

197. Jedamzik, K., Katalinić, V., Olinto, A.V.: PRL 85, 700 (2000). https://doi.org/10.1103/PhysRevLett. 85.700

198. Sethi, S.K., Subramanian, K.: MNRAS 356, 778 (2005). https://doi.org/10.1111/j.1365-2966. 2004.08520.x

199. Kunze, K.E.: E. Komatsu, JCAP 1, 009 (2014). https://doi.org/10.1088/1475-7516/2014/01/009

200. Wagstaff, J.M., Banerjee, R.: Phys. Rev. D92(12) 123004. https://doi.org/10.1103/PhysRevD. $92.123004(2015)$

201. Pani, P., Loeb, A.: Phys. Rev. D88(4) 041301. https://doi.org/10.1103/PhysRevD.88.041301 (2013)

202. Clesse, S., García-Bellido, J.: Phys. Rev. D92(2) 023524. https://doi.org/10.1103/PhysRevD. 92.023524 (2015)

203. Nakama, T., Carr, B., Silk, J.: Phys. Rev. D D97(4) 043525. https://doi.org/10.1103/PhysRevD. 97.043525 (2018)

204. Bird, S., et al.: Phys. Rev. Lett 116(20), 201301 (2016). https://doi.org/10.1103/PhysRevLett.116. 201301

205. Sasaki, M., et al.: Phys. Rev. Lett. 117(6), 061101 (2016). https://doi.org/10.1103/PhysRevLett.121. 059901, https://doi.org/10.1103/PhysRevLett.117.061101

206. Sasaki, M., et al.: Class. Quant. Grav. 35(6), 063001 (2018). https://doi.org/10.1088/1361-6382/ aaa7b4

207. Abbott, B.P., et al.: Phys. Rev. Lett. 116(6), 061102 (2016). https://doi.org/10.1103/PhysRevLett. 116.061102 
208. Niikura, H. et al.: Nat. Astron. 3(6), 524 (2019). https://doi.org/10.1038/s41550-019-0723-1

209. Carr, B., Kuhnel, F., Sandstad, M.: Phys. Rev. D94(8) 083504. https://doi.org/10.1103/PhysRevD.94. 083504 (2016)

210. Kawasaki, M., Kusenko, A., Yanagida, T.T.: Phys. Lett. B711, 1 (2012). https://doi.org/10.1016/ j.physletb.2012.03.056

211. Kohri, K., Nakama, T., Suyama, T.: Phys. Rev. D90, 083514 (2014). https://doi.org/10.1103/ PhysRevD.90.083514

212. Kawasaki, M., Murai, K.: (2019)

213. Carr, B.J.: Astrophys. J. 201, 1 (1975). https://doi.org/10.1086/153853

214. Harada, T., et al.: Phys. Rev. D 88(8), 084051 (2013). https://doi.org/10.1103/PhysRevD.88.084051, https://doi.org/10.1103/PhysRevD.89.029903

215. Harada, T., et al.: Phys. Rev. D96(8), 083517 (2017). https://doi.org/10.1103/PhysRevD.99.069904, https://doi.org/10.1103/PhysRevD.96.083517

216. Inomata, K., et al.: Phys. Rev. D 97(4), 043514 (2018). https://doi.org/10.1103/PhysRevD.97.043514

217. Lyth, D.H.: arXiv:1107.1681 (2011)

218. Kawasaki, M., Kitajima, N., Yanagida, T.T.: Phys. Rev. D87(6) 063519. https://doi.org/10.1103/ PhysRevD.87.063519 (2013)

219. Kohri, K., Lin, C., Matsuda, T.: Phys. Rev. D87(10) 103527. https://doi.org/10.1103/PhysRevD.87. 103527 (2013)

220. Frampton, P.H., Kawasaki, M., Takahashi, F., Yanagida, T.T.: JCAP 1004, 023 (2010). https:// doi.org/10.1088/1475-7516/2010/04/023

221. Josan, A.S., Green, A.M., Malik, K.A.: Phys. Rev. 103520, D79 (2009). https://doi.org/10.1103/ PhysRevD.79.103520

222. Stöcker, P., Krämer, M., Lesgourgues, J., Poulin, V.: JCAP 1803(03), 018 (2018). https://doi.org/ 10.1088/1475-7516/2018/03/018

223. Ali-Haïmoud, Y., Kamionkowski, M.: Phys. Rev. D95(4) 043534. https://doi.org/10.1103/ PhysRevD.95.043534 (2017)

224. Poulin, V. et al.: Phys. Rev. D96(8) 083524. https://doi.org/10.1103/PhysRevD.96.083524 (2017)

225. Jeong, D., et al.: Phys. Rev. Lett 113(6), 061301 (2014). https://doi.org/10.1103/PhysRevLett.113. 061301

226. Nakama, T., Suyama, T., Yokoyama, J.: Phys. Rev. Lett. 113, 061302 (2014). https://doi.org/ 10.1103/PhysRevLett.113.061302

227. Inomata, K., Kawasaki, M., Tada, Y.: Phys. Rev. D94(4) 043527. https://doi.org/10.1103/PhysRevD. 94.043527 (2016)

228. Nakama, T., Carr, B., Silk, J.: Phys. Rev. D97(4) 043525. https://doi.org/10.1103/PhysRevD.97. 043525 (2018)

229. Inman, D., Ali-Haïmoud, Y.: Phys. Rev. D 100(8), 083528 (2019). https://doi.org/10.1103/ PhysRevD.100.083528

230. Tashiro, H., Sugiyama, N.: Phys. Rev. D78, 023004 (2008). https://doi.org/10.1103/PhysRevD.78. 023004

231. Peccei, R.D., Quinn, H.R.: Phys. Rev. Lett. 38, 1440 (1977). https://doi.org/10.1103/PhysRevLett. 38.1440

232. Weinberg, S.: Phys. Rev. Lett. 40, 223 (1978). https://doi.org/10.1103/PhysRevLett.40.223

233. Wilczek, F.: Phys. Rev. Lett. 40, 279 (1978). https://doi.org/10.1103/PhysRevLett.40.279

234. Svrcek, P.: E. Witten. JHEP 06, 051 (2006). https://doi.org/10.1088/1126-6708/2006/06/051

235. Arvanitaki, A. et al.: Phys. Rev. D 123530, 81 (2010). https://doi.org/10.1103/PhysRevD.81.123530

236. Acharya, B.S., Bobkov, K., Kumar, P.: JHEP 11, 105 (2010). https://doi.org/10.1007/JHEP11 (2010) 105

237. Graham, P.W. et al.: Annu. Rev. Nucl. Part. Sci. 65, 485 (2015). https://doi.org/10.1146/annurevnucl-102014-022120

238. Ruz, J., Vogel, J.K., Pivovaroff, M.: J. Phys. Proced. 61, 153 (2015). https://doi.org/10.1016/ j.phpro.2014.12.025

239. Bastidon, N.: I. for the ALPS collaboration (2015)

240. Majorovits, B. et al.: (2016)

241. Asztalos, S.J., et al.: Phys. Rev. Lett 104(4), 041301 (2010). https://doi.org/10.1103/PhysRevLett. 104.041301

242. Budker, D., et al.: Phys. Rev. X 4(2), 021030 (2014). https://doi.org/10.1103/PhysRevX.4.021030 
243. Hu, W., Barkana, R., Gruzinov, A.: Phys. Rev. Lett. 85, 1158 (2000). https://doi.org/10.1103/ PhysRevLett.85.1158

244. Park, C.G., Hwang, J.C., Noh, H.: Phys. Rev. D 86, 083535 (2012). https://doi.org/10.1103/ PhysRevD.86.083535

245. Hlozek, R., Grin, D., Marsh, D.J.E., Ferreira, P.G.: Phys. Rev. D 91(10), 103512 (2015). https://doi.org/10.1103/PhysRevD.91.103512

246. Hlozek, R., et al.: Phys. Rev. D 95(12), 123511 (2017). https://doi.org/10.1103/PhysRevD.95.123511

247. Hlozek, R., Marsh, D.J.E., Grin, D.: MNRAS 476(3), 3063 (2018). https://doi.org/10.1093/ mnras/sty 271

248. Sikivie, P.: Phys. Rev. Lett. 51, 1415 (1983). https://doi.org/10.1103/PhysRevLett.51.1415

249. Raffelt, G., Stodolsky, L.: Phys. Rev. D 37, 1237 (1988). https://doi.org/10.1103/PhysRevD.37.1237

250. Anselm, A.A.: Phys. Rev. D 37, 2001 (1988). https://doi.org/10.1103/PhysRevD.37.2001

251. Cordes, J.M., Lazio, T.J.W.: (2002)

252. Jansson, R., Farrar, G.R.: ApJ 757(1), 14 (2012). https://doi.org/10.1088/0004-637X/757/1/14

253. Jansson, R., Farrar, G.R.: ApJL 761(1), L11 (2012). https://doi.org/10.1088/2041-8205/761/1/L11

254. Mukherjee, S., Spergel, D.N., Khatri, R., Wandelt, B.D.: JCAP 02, 032 (2020). https://doi.org/ 10.1088/1475-7516/2020/02/032

255. Delabrouille, J., Abitbol, M.H., Aghanim, N., Ali-Haimoud, Y., Alonso, D., Alvarez, M., Banday, A.J., Bartlett, J.G., Baselmans, J., Basu, K., Battaglia, N., Bermejo Climent, J.R., Bernal, J.L., Béthermin, M., Bolliet, B., Bonato, M., Bouchet, F.R., Breysse, P.C., Burigana, C., Cai, Z.Y., Chluba, J., Churazov, E., Dannerbauer, H., De Bernardis, P., De Zotti, G., Di Valentino, E., Dimastrogiovanni, E., Endo, A., Erler, J., Ferraro, S., Finelli, F., Fixsen, D., Hanany, S., Hart, L., Hernandez-Monteagudo, C., Hill, J.C., Hotinli, S.C., Karatsu, K., Karkare, K., Keating, G.K., Khabibullin, I., Kogut, A., Kohri, K., Kovetz, E.D., Lagache, G., Lesgourgues, J., Madhavacheril, M., Maffei, B., Mandolesi, N., Martins, C., Masi, S., Mather, J., Melin, J.B., Moradinezhad Dizgah, A., Mroczkowski, T., Mukherjee, S., Nagai, D., Negrello, M., Palanque-Delabrouille, N., Paoletti, D., Patil, S.P., Piacentini, F., Raghunathan, S., Ravenni, A., Remazeilles, M., Revéret, V., Rodriguez, L., Rotti, A., Rubino Martin, J.A., Sayers, J., Scott, D., Silk, J., Silva, M., Souradeep, T., Sugiyama, N., Sunyaev, R., Switzer, E.R., Tartari, A., Trombetti, T., Zubeldia, I.: arXiv:1909.01591 (2019)

256. Mukherjee, S., Khatri, R., Wandelt, B.D.: JCAP 1906(06), 031 (2019). https://doi.org/10.1088/ $1475-7516 / 2019 / 06 / 031$

257. Rybicki, G.B., dell'Antonio, I.P.: ApJ 427, 603 (1994). https://doi.org/10.1086/174170

258. Dubrovich, V.K., Stolyarov, V.A.: A\&A 302, 635 (1995)

259. Kholupenko, E.E. et al.: Gravit. Cosmol. 11, 161 (2005)

260. Wong, W.Y., Seager, S., Scott, D.: MNRAS 367, 1666 (2006). https://doi.org/10.1111/j.1365-2966. 2006.10076.x

261. Rubiño-Martín, J.A., Chluba, J., Sunyaev, R.A.: MNRAS 371, 1939 (2006). https://doi.org/10.1111/ j.1365-2966.2006.10839.x

262. Chluba, J., Sunyaev, R.A.: A\&A 446, 39 (2006). https://doi.org/10.1051/0004-6361:20053988

263. Rubiño-Martín, J.A., Chluba, J., Sunyaev, R.A.: A\&A 485, 377 (2008). https://doi.org/10.1051/ 0004-6361:20078993

264. Ali-Haïmoud, Y.: Phys. Rev. D 87(2), 023526 (2013). https://doi.org/10.1103/PhysRevD.87.023526

265. Chluba, J., Sunyaev, R.A.: A\&A 478, L27 (2008). https://doi.org/10.1051/0004-6361:20078200

266. Zeldovich, Y.B., Kurt, V.G., Syunyaev, R.A.: ZhETF 55, 278 (1968)

267. Peebles, P.J.E.: ApJ 153, 1 (1968)

268. Sunyaev, R.A., Zeldovich, Y.B.: ApSS 7, 3 (1970)

269. Peebles, P.J.E., Yu, J.T.: ApJ 162, 815 (1970). https://doi.org/10.1086/150713

270. Hu, W., Scott, D., Sugiyama, N., White, M.: Phys. Rev. D 52, 5498 (1995)

271. Lewis, A., Weller, J., Battye, R.: MNRAS 373, 561 (2006). https://doi.org/10.1111/j.1365-2966. 2006.10983.x

272. Rubiño-Martín, J.A., Chluba, J., Fendt, W.A., Wandelt, B.D.: MNRAS 403, 439 (2010). https://doi.org/10.1111/j.1365-2966.2009.16136.x

273. Shaw, J.R.: J. Chluba, MNRAS 415, 1343 (2011). https://doi.org/10.1111/j.1365-2966.2011.18782.x

274. Chluba, J., Vasil, G.M., Dursi, L.J.: MNRAS 407, 599 (2010). https://doi.org/10.1111/j.1365-2966. 2010.16940.x

275. Desjacques, V., Chluba, J., Silk, J., de Bernardis, F., Doré, O.: MNRAS 451, 4460 (2015). https://doi.org/10.1093/mnras/stv1291 
276. Sathyanarayana Rao, M., Subrahmanyan, R., Udaya Shankar, N.: J. Chluba. ApJ 810, 3 (2015). https://doi.org/10.1088/0004-637X/810/1/3

277. Hart, L., Rotti, A., Chluba, J.: arXiv:2006.04826 (2020)

278. Sunyaev, R.A., Zeldovich, Y.B.: A\&A 20, 189 (1972)

279. Hu, W., Scott, D., Silk, J.: Phys. Rev. D 49, 648 (1994). https://doi.org/10.1103/PhysRevD.49.648

280. Cen, R., Ostriker, J.P.: ApJ 514, 1 (1999). https://doi.org/10.1086/306949

281. Miniati, F., Ryu, D., Kang, H., Jones, T.W., Cen, R., Ostriker, J.P.: ApJ 542, 608 (2000). https://doi.org/10.1086/317027

282. Oh, S.P., Cooray, A., Kamionkowski, M.: MNRAS 342, L20 (2003). https://doi.org/10.1046/ j.1365-8711.2003.06708.x

283. Khatri, R., Sunyaev, R.: JCAP 8, 013 (2015). https://doi.org/10.1088/1475-7516/2015/08/013

284. Hill, J.C., et al.: Phys. Rev. Lett 115(26), 261301 (2015). https://doi.org/10.1103/PhysRevLett.115. 261301

285. Battaglia, N., Bond, J.R., Pfrommer, C., Sievers, J.L., Sijacki, D.: ApJ 725, 91 (2010). https:// doi.org/10.1088/0004-637X/725/1/91

286. Le Brun, A.M.C., McCarthy, I.G., Schaye, J., Ponman, T.J.: MNRAS 441(2), 1270 (2014). https://doi.org/10.1093/mnras/stu608

287. Wright, E.L.: ApJ 232, 348 (1979). https://doi.org/10.1086/157294

288. Rephaeli, Y.: ApJ 445, 33 (1995). https://doi.org/10.1086/175669

289. Sazonov, S.Y., Sunyaev, R.A.: ApJ 508, 1 (1998). https://doi.org/10.1086/306406

290. Itoh, N., Kohyama, Y., Nozawa, S.: ApJ 502, 7 (1998). https://doi.org/10.1086/305876

291. Challinor, A., Lasenby, A.: ApJ 499, 1 (1998). https://doi.org/10.1086/305623

292. Chluba, J., Nagai, D., Sazonov, S., Nelson, K.: MNRAS 426, 510 (2012). https://doi.org/10.1111/ j.1365-2966.2012.21741.x

293. Kay, S.T., Powell, L.C., Liddle, A.R., Thomas, P.A.: MNRAS 386, 2110 (2008). https://doi.org/ 10.1111/j.1365-2966.2008.13183.x

294. Lee, E., Chluba, J., Kay, S.T., Barnes, D.J.: MNRAS 493(3), 3274 (2020). https://doi.org/10.1093/ mnras/staa450

295. Zeldovich, Y.B., Illarionov, A.F., Sunyaev, R.A.: SJETP 35, 643 (1972)

296. Shimon, M., Rephaeli, Y.: ApJ 575, 12 (2002). https://doi.org/10.1086/341268

297. Colafrancesco, S., Marchegiani, P., Palladino, E.: A\&A 397, 27 (2003). https://doi.org/10.1051/ 0004-6361:20021199

298. Colafrancesco, S., Profumo, S., Ullio, P.: A\&A 455, 21 (2006). https://doi.org/10.1051/00046361:20053887

299. Stebbins, A., Silk, J.: ApJ 300, 1 (1986). https://doi.org/10.1086/163778

300. Danese, L., Burigana, C.: Theor. Aspects CMB Spectrum 429, 28 (1994). https://doi.org/10.1007/3540-57755-6_29

301. Cooray, A., Furlanetto, S.R.: ApJL 606(1), L5 (2004). https://doi.org/10.1086/421241

302. Ponente, P.P., Diego, J.M., Sheth, R.K., Burigana, C., Knollmann, S.R., Ascasibar, Y.: MNRAS 410, 2353 (2011). https://doi.org/10.1111/j.1365-2966.2010.17611.x

303. Trombetti, T., Burigana, C.: MNRAS 437, 2507 (2014). https://doi.org/10.1093/mnras/stt2063

304. Fixsen, D.J. et al.: ApJ 508, 123 (1998). https://doi.org/10.1086/306383

305. Serra, P., Lagache, G., Doré, O., Pullen, A., White, M.: A\&A 570, A98 (2014). https://doi.org/ 10.1051/0004-6361/201423958

306. Kovetz, E.D. et al.: arXiv e-prints (2017)

307. Kovetz, E. et al.: BAAS 51(3), 101 (2019)

308. Carilli, C.L., Walter, F.: ARA\&A 51, 105 (2013). https://doi.org/10.1146/annurev-astro-082812140953

309. Pullen, A.R., Serra, P., Chang, T.C., Doré, O., Ho, S.: MNRAS 478, 1911 (2018). https://doi.org/10.1093/mnras/sty1243

310. Yang, S., Pullen, A.R., Switzer, E.R.: arXiv e-prints (2019)

311. Padmanabhan, H.: MNRAS, 1857. https://doi.org/10.1093/mnras/stz1878 (2019)

312. Mashian, N., Loeb, A., Sternberg, A.: MNRAS 458(1), L99 (2016). https://doi.org/10.1093/mnrasl/ slw027

313. Serra, P., Doré, O., Lagache, G.: ApJ 833, 153 (2016). https://doi.org/10.3847/1538-4357/833/2/153

314. Switzer, E.R.: ApJ 838, 82 (2017). https://doi.org/10.3847/1538-4357/aa6576

315. Switzer, E.R., Anderson, C.J., Pullen, A.R., Yang, S.: ApJ 872, 82 (2019). https://doi.org/10.3847/ 1538-4357/aaf9ab 
316. Moradinezhad Dizgah, A., Keating, G.K.: ApJ 872(2), 126 (2019). https://doi.org/10.3847/15384357/aafd36

317. Decarli, R., Walter, F., Gónzalez-López, J., Aravena, M., Boogaard, L., Carilli, C., Cox, P., Daddi, E., Popping, G., Riechers, D., Uzgil, B., Weiss, A., Assef, R.J., Bacon, R., Bauer, F.E., Bertoldi, F., Bouwens, R., Contini, T., Cortes, P.C., da Cunha, E., Díaz-Santos, T., Elbaz, D., Inami, H., Hodge, J., Ivison, R., Le Fèvre, O., Magnelli, B., Novak, M., Oesch, P., Rix, H.W., Sargent, M.T., Smail, I., Swinbank, A.M., Somerville, R.S., van der Werf, P., Wagg, J., Wisotzki, L.: ApJ 882(2), 138 (2019). https://doi.org/10.3847/1538-4357/ab30fe

318. Loeb, A.: ApJL 555, L1 (2001). https://doi.org/10.1086/321730

319. Zaldarriaga, M., Loeb, A.: ApJ 564, 52 (2002). https://doi.org/10.1086/324149

320. Basu, K., Hernández-Monteagudo, C., Sunyaev, R.A.: A\&A 416, 447 (2004). https://doi.org/ 10.1051/0004-6361:20034298

321. Rubiño-Martín, J.A., Hernández-Monteagudo, C., Sunyaev, R.A.: A\&A 438, 461 (2005). https:// doi.org/10.1051/0004-6361:20052939

322. Hernández-Monteagudo, C., Sunyaev, R.A.: MNRAS 359, 597 (2005). https://doi.org/10.1111/ j.1365-2966.2005.08929.x

323. Hernández-Monteagudo, C., Verde, L., Jimenez, R.: ApJ 653, 1 (2006). https://doi.org/10.1086/ 508529

324. Hernández-Monteagudo, C., Rubiño-Martín, J.A., Sunyaev, R.A.: MNRAS 380, 1656 (2007). https://doi.org/10.1111/j.1365-2966.2007.12218.x

325. Hernández-Monteagudo, C., Maio, U., Ciardi, B., Sunyaev, R.A.: arXiv e-prints (2017)

326. Yu, Q., Spergel, D.N., Ostriker, J.P.: ApJ 558, 23 (2001). https://doi.org/10.1086/322482

327. Lewis, A.: JCAP 8, 053 (2013). https://doi.org/10.1088/1475-7516/2013/08/053

328. Righi, M., Hernández-Monteagudo, C., Sunyaev, R.A.: A\&A 489, 489 (2008). https://doi.org/ 10.1051/0004-6361:200810199

329. Hernández-Monteagudo, C., Haiman, Z., Jimenez, R., Verde, L.: ApJL 660, L85 (2007). https://doi.org/10.1086/518090

330. Gong, Y. et al.: ApJ 745, 49 (2012). https://doi.org/10.1088/0004-637X/745/1/49

331. Aghanim, N. et al.: ESA F-class mission proposal (2019)

332. Abitbol, M.H., Chluba, J., Hill, J.C., Johnson, B.R.: MNRAS 471, 1126 (2017). https://doi.org/ $10.1093 / \mathrm{mnras} / \mathrm{stx} 1653$

333. Masi, S. et al.: Memorie della Soc. Astron. Italian. 74, 96 (2003)

334. Schillaci, A. et al.: A\&A 565, A125 (2014). https://doi.org/10.1051/0004-6361/201423631

335. Kogut, A. et al.: New Astron. Rev. 50, 925 (2006). https://doi.org/10.1016/j.newar.2006.09.023

336. Seiffert, M. et al.: ApJ 734, 6 (2011). https://doi.org/10.1088/0004-637X/734/1/6

337. Maffei, B. et al.: Balloon proposal to CNES (2019)

338. Chluba, J., Hill, J.C., Abitbol, M.H.: MNRAS 472, 1195 (2017). https://doi.org/10.1093/mnras/ stx 1982

339. Remazeilles, M., Chluba, J.: MNRAS 478, 807 (2018). https://doi.org/10.1093/mnras/sty1034

340. Remazeilles, M. et al.: JCAP 4, 023 (2018). https://doi.org/10.1088/1475-7516/2018/04/023

341. Rotti, A., Chluba, J.: arXiv:2006.02458 (2020)

342. Remazeilles, M., Rotti, A., Chluba, J.: arXiv:2006.08628 (2020)

343. Silk, J.: Nature 553(7686), 6 (2018). https://doi.org/10.1038/d41586-017-08941-8

344. Righi, M., Hernández-Monteagudo, C., Sunyaev, R.A.: A\&A 478, 685 (2008). https://doi.org/ 10.1051/0004-6361:20078207

345. Planck Collaboration (2019). arXiv e-prints

Publisher's note Springer Nature remains neutral with regard to jurisdictional claims in published maps and institutional affiliations. 


\section{Affiliations}

J. Chluba ${ }^{1}$ (D) M. H. Abitbol ${ }^{2}$ - N. Aghanim ${ }^{3}$ - Y. Ali-Haïmoud ${ }^{4}$ - M. Alvarez Al6 $^{5}$. K. Basu ${ }^{7}$. B. Bolliet ${ }^{1}$. C. Burigana ${ }^{8,9,10}$. P. de Bernardis ${ }^{11,12}$. J. Delabrouille ${ }^{13,14} \cdot$ E. Dimastrogiovanni ${ }^{15} \cdot$ F. Finelli $^{16}$. D. Fixsen ${ }^{17}$. L. Hart ${ }^{1}$. C. Hernández-Monteagudo ${ }^{18}$. J. C. Hill ${ }^{19,20}$ - A. Kogut ${ }^{21}$. K. Kohri' ${ }^{22}$. J. Lesgourgues ${ }^{23}$. B. Maffei ${ }^{3}$. J. Mather ${ }^{21}$. S. Mukherjee 24,25,26 . S. P. Patil ${ }^{27}$ - A. Ravenni ${ }^{1}$ - M. Remazeilles ${ }^{1}$ - A. Rotti ${ }^{1}$. J. A. Rubiño-Martin ${ }^{28,29}$. J. Silk 30,31 - R. A. Sunyaev ${ }^{19,32,33}$ - E. R. Switzer 21

1 Jodrell Bank Centre for Astrophysics, Department of Physics \& Astronomy, The University of Manchester, Manchester M13 9PL, UK

2 University of Oxford, Denys Wilkinson Building, Keble Road, Oxford, OX1 3RH, UK

3 Institut d'Astrophysique Spatiale (IAS), CNRS (UMR8617), Université Paris-Sud, Batiment 121, 91405 Orsay, France

4 Center for Cosmology and Particle Physics, Department of Physics, New York University, New York, NY 10003, USA

5 Berkeley Center for Cosmological Physics, University of California, Berkeley, CA 94720, USA

6 Lawrence Berkeley National Laboratory, One Cyclotron Road, Berkeley, CA 94720, USA

7 Argelander-Institut für Astronomie, Universität Bonn, Auf dem Hügel 71, 53121 Bonn, Germany

8 INAF, Istituto di Radioastronomia, Via Piero Gobetti 101, I-40129 Bologna, Italy

9 Dipartimento di Fisica e Scienze della Terra, Università di Ferrara, Via Giuseppe Saragat 1, I-44122 Ferrara, Italy

10 INFN, Sezione di Bologna, Via Irnerio 46, I-40126 Bologna, Italy

11 Physics department, Sapienza University of Rome, Piazzale Aldo Moro 5, 00185, Rome, Italy

12 INFN sezione di Roma, P.le A. Moro 2, 00815 Roma, Italy

13 Laboratoire Astroparticule et Cosmologie, CNRS/IN2P3, 10, rue Alice Domon et Léonie Duquet, 75205 Paris Cedex 13, France

14 Département d'Astrophysique, CEA Saclay DSM/Irfu, 91191 Gif-sur-Yvette, France School of Physics, The University of New South Wales, Sydney NSW 2052, Australia INAF - Osservatorio di Astrofisica e Scienza dello Spazio, Via Gobetti 101, I-40129 Bologna, Italy Department of Astronomy, University of Maryland, College Park, MD 20742-2421, USA

Centro de Estudios de Física del Cosmos de Aragón (CEFCA), Plaza San Juan, 1, planta 2, E-44001, Teruel, Spain

19 Institute for Advanced Study, Princeton, NJ 08540, USA

20 Center for Computational Astrophysics, Flatiron Institute, 162 5th Avenue, New York, NY 10010, USA

21 NASA/GSFC, Mail Code: 665, Greenbelt, MD 20771, USA

22 KEK and Sokendai, Tsukuba 305-0801, Japan, and Kavli IPMU, U. of Tokyo, Kashiwa 277-8582, Japan

23 Institute for Theoretical Particle Physics and Cosmology (TTK), RWTH Aachen University, 52056 Aachen, Germany

24 Institut d'Astrophysique de Paris, Sorbonne Université, CNRS, UMR 7095, 98 bis bd Arago, 75014 Paris, France

25 Institut Lagrange de Paris, Sorbonne Universites, 98 bis Boulevard Arago, 75014 Paris, France

26 Gravitation Astroparticle Physics Amsterdam (GRAPPA), University of Amsterdam, Science Park 904, 1090 GL Amsterdam, The Netherlands 
27 Niels Bohr International Academy and Discovery Center, Blegdamsvej 17, 2100 Copenhagen, Denmark

28 Instituto de Astrofísica de Canarias, E-38200 La Laguna, Tenerife, Spain

29 Departamento de Astrofísica, Universidad de La Laguna, E-38206 La Laguna, Tenerife, Spain

30 Department of Physics and Astronomy, The Johns Hopkins University, 3701 San Martin Drive, Baltimore MD 21218, USA

31 Department of Physics and Beecroft Inst. for PAC, University of Oxford, 1 Keble Road, Oxford, OX1 3RH, UK

32 Max-Planck-Institut für Astrophysik, Karl-Schwarzschild Str. 1, 85741 Garching, Germany

33 Space Research Institute (IKI) of the Russian Academy of Sciences, 84/32, Profsoyuznaya str., Moscow, 117810, Russia 